\title{
ON MAXIMAL SYMPLECTIC PARTIAL SPREADS
}

\author{
W. M. KANTOR
}

AbStRACT. New types of maximal symplectic partial spreads are constructed.

\section{INTRODUCTION}

Since very few papers concern maximal symplectic partial spreads in dimension $>4[\mathrm{Gr}$, this paper will focus on those dimensions. The largest and most obvious type of maximal partial spread of a $2 n$-dimensional symplectic $\mathbb{F}_{q}$-space is a spread, of size $q^{n}+1$, which we will not consider here. (However, there are relatively few known types of symplectic spreads, see [Ka3] for a survey as of 2012.)

On the other hand, when $n$ is even Grassl $\mathrm{Gr}$ ] initially conjectured that the smallest possible size of a maximal symplectic partial spread is $q^{n / 2}+1$, and he provided examples of this size for all even $q$ and $n$. However, when $2 n=8$ the conjecture is not correct $\mathrm{Gr}$. Families of counterexamples using Suzuki-Tits ovoids are in Section 7.4. It still seems plausible that Grassl's conjecture may be correct if $2 n>8$ or if $q$ is odd. Thus far all counterexamples to this conjecture have size greater than $q^{n / 2} / 2$.

Most of our examples are based on standard properties of orthogonal and symplectic spaces, involving either orthogonal spreads or the standard method for obtaining them (Sections 4 , 5 and 6), or partial $\mathrm{O}^{+}(8, q)$-ovoids and triality (Section 7). Approximately half of this paper is concerned with spaces of dimension 4 or 8 , where we can use points as crutches: the Klein correspondence in dimension 4 [Ta, p. 196] and triality in dimension 8 [Ti1] turn sets of points into sets of subspaces (of dimension 2 or 4 ). In dimension $>4$ our results are summarized in Table 1 the pairs of dimensions of the form $4 n, 4 n-2$ arise from orthogonal partial spreads and are explained in Section 6.

Maximal symplectic partial spreads have a straightforward use in Quantum Physics for finding sets of mutually unbiased bases [MBGW, Gr] (e.g., by plugging into [Ka3, Eqs. (3.2) or (3.4)] in order to obtain sets of complex vectors).

There are tables of computer-generated sizes of maximal symplectic partial spreads in $\mathbb{F}_{q}^{2 n}$ for very small $n$ and $q$ [CDFS, Gr. A few of these are special cases of constructions given here. However, since these tables contain integer intervals that consist of sizes of these partial spreads, it is clear that new types of construction techniques are needed in all dimensions.

\section{BACKGROUND}

The letter $q$ will always denote a prime power, while $n, m, k, s$ and $i$ will be integers. 
TABLE 1. Maximal symplectic partial spreads: dimension $\geq 6$ over $\mathbb{F}_{q}$

\begin{tabular}{|c|c|c|c|c|}
\hline dimensions & parity of $q$ & Size & Restrictions & Theorems \\
\hline $4 m$ & arbitrary & $q^{2 m}-q^{m}+(2, q-1)$ & & 3.1 \\
\hline $4 m k, 4 m k-2$ & even & $q^{2 m k-k}+1$ & $m>(k+1) / 2$ & $4.5,6.3$ \\
\hline $4 k, 4 k-2$ & even & $q^{k}+1^{\mathrm{a}}$ & & $5.2,6.2$ \\
\hline $4 k$ & even & $2 q^{k}+1$ & & 5.2 \\
\hline 8 and 6 & even & $q^{3}-q^{2}+1$ & $q \geq 4$ & 7.27 .15 \\
\hline 8 and 6 & even & $n_{s}{ }^{\mathrm{b}}$ & $1 \leq s \leq q / 5$ & $7.3,7.15$ \\
\hline 8 and 6 & even & $n_{4}-1$ & $q \geq 16$ & $7.3,7.15$ \\
\hline 8 and 6 & even & $q^{2}+1$ & & 7.77 .15 \\
\hline 8 and 6 & even & $2 q^{2}+1$ & & $7.10,7.15$ \\
\hline 8 and 6 & even & $q^{2}+q+1$ & $q=2^{2 e+1}>2$ & $7.11,7.15$ \\
\hline 8 and 6 & even & $q^{2}-q+1$ & $q=2^{2 e+1}>2$ & $7.13,7.15$ \\
\hline 8 and 6 & even & $q^{2}-s q+2 s-1$ & $\begin{array}{l}q=2^{2 e+1}>2 \\
1<s \leq 2^{e}-1\end{array}$ & $7.14,7.15$ \\
\hline 6 & arbitrary & $q^{3}-q^{2}+1$ & & 8.1 \\
\hline
\end{tabular}

${ }^{\mathrm{a}}$ This corresponds to the excluded possibility $m=1$ in dimensions $4 m k, 4 m k-2$.

${ }^{\mathrm{b}} n_{s}=q^{3}-s q^{2}+(s-1)(q+2)+\left(\begin{array}{c}s \\ 2\end{array}\right)(q-2)+1$

See $\mathrm{Ta}$ ] for the standard properties of the symplectic and orthogonal vector spaces used here. We name geometries using their isometry groups. We will be concerned with singular vectors and totally singular (t.s.) subspaces of orthogonal spaces, and totally isotropic (t.i.) subspaces of symplectic spaces. A subspace of an orthogonal space is anisotropic if it contains no nonzero singular vector - and hence has dimension $\leq 2$. In characteristic 2 , an orthogonal vector space is also a symplectic space, and t.s. subspaces are also t.i. subspaces.

Types of maximal t.s. subspaces. The $n$-dimensional t.s. subspaces of an $\mathrm{O}^{+}(2 n, q)$-space are of two types, with two such subspaces of the same type if and only if their intersection has dimension $\equiv n(\bmod 2)$. Each t.s. $n-1$-space is contained in one member of each type. Since we will be concerned with subspaces intersecting in $0, n$ will be even.

A triality map for an $\mathrm{O}^{+}(8, q)$-space [Ti1] permutes the t.s. subspaces, sending singular points to a type of t.s. 4 -spaces and non-perpendicular pairs of points to pairs of 4-spaces having zero intersection.

Partial ovoids and partial spreads. A partial ovoid of an orthogonal space is a set $\Omega$ of t.s. points such that each maximal t.s. subspace contains at most one point in the set; $\Omega$ is an ovoid if it meets every such subspace. A partial spread in a 
$2 n$-dimensional vector space $V$ is a set $\Sigma$ of $n$-spaces any two of which have only 0 in common; $\Sigma$ is a spread if every vector is in a member of $\Sigma$. If $V$ is a $2 n$-dimensional symplectic or orthogonal vector space, a symplectic or orthogonal partial spread $\Sigma$ is a partial spread consisting of t.i. or t.s. $n$-spaces; $\Sigma$ is a symplectic or orthogonal spread if every vector or every singular vector is in a member of $\Sigma$. This note concerns maximal symplectic or orthogonal partial spreads: maximal with respect to inclusion. In some situations we will even obtain symplectic maximal partial spreads: maximal partial spreads that happen to be symplectic.

Two symplectic partial spreads are equivalent if there is a semilinear automorphism of the symplectic geometry sending one partial spread to the other. If $\Sigma$ is a set of subspaces of an $\operatorname{Sp}(2 n, q)$-space, then $\operatorname{Sp}(2 n, q)_{\Sigma}$ is its set-stabilizer in the symplectic group $\operatorname{Sp}(2 n, q)$. There are similar definitions for orthogonal spaces and for the automorphism group of a symplectic or orthogonal partial spread.

\section{Maximal Partial $\operatorname{Sp}(4 m, q)$-SPREAdS}

Our most general result is the following

Theorem 3.1. For any $q$ and $m \geq 1$, an $\mathrm{Sp}(4 m, q)$-space has a maximal symplectic partial spread of size $q^{2 m}-q^{m}+(2, q-1)$.

We begin with notation. Let $F=\mathbb{F}_{q^{2 m}} \supset E=\mathbb{F}_{q^{m}} \supset K=\mathbb{F}_{q}$, with trace map $T: F \rightarrow K$, so that $T(x y)$ is a nondegenerate symmetric $K$-bilinear form on $F$. By dimensions, $\{x \in F \mid T(x E)=0\}=\theta E$ for some $\theta \in F$.

Equip the $K$-space $V=F^{2}$ with the nondegenerate alternating $K$-bilinear form $f\left((x, y),\left(x^{\prime}, y^{\prime}\right)\right):=T\left(x y^{\prime}\right)-T\left(x^{\prime} y\right)$.

Let $\Sigma$ be the desarguesian symplectic spread of $V$ consisting of the t.i. 2-spaces $[x=0]$ and $[y=a x]$ for $a \in F$. Let $Z_{\star}$ be the t.i. $2 m$-space $(E, \theta E)=E \oplus \theta E$ (t.i. since $T(E \theta E)=0)$.

Let $\Sigma_{\star} \subset \Sigma$ consist of the members of $\Sigma$ met nontrivially by $Z_{\star}$ (namely, the $2 m$-spaces $[x=0]$ and $[y=a \theta x]$ for $a \in E)$. We need information concerning some transversals of $\Sigma_{\star}$ :

Lemma 3.2. There are exactly $(2, q-1)$ t.i. $2 m$-spaces of the $\operatorname{Sp}(4 m, q)$-space $V$ that meet each member of $\Sigma_{\star}$ in an $m$-space. If there are two such subspaces then they intersect in 0 .

Proof. If $Z$ is such a subspace let $Z \cap[y=0]=(U, 0)$ and $Z \cap[x=0]=(0, W)$ for $m$-dimensional $K$-subspaces $U$ and $W$ of $F$. Since $Z=(U, 0)+(0, W)$ is t.i. we have $T(U W)=0$.

Since $Z \cap[y=a \theta x]$ (for $a \in E$ ) consists of the vectors $(u, a \theta u$ ) with $u \in U$, we see that $W=\theta U$ (using $a=1$ ) and $W$ is closed under multiplication by elements of $E$. Then $W$ is an $E$-subspace of $F$. Let $U=\alpha E, \alpha \in F^{*}$, so that $W=\theta \alpha E$. Then $0=T(U W)=T(\alpha \theta \alpha E)$, so that $\alpha^{2} \theta \in \theta E$. Thus, there are $(2, q-1)$ choices for the coset $\alpha F^{*} \in F^{*} / E^{*}$, and hence also $(2, q-1)$ choices for $Z=(U, W)=(\alpha E, \theta \alpha E)$.

This argument reverses: if the coset $\alpha E^{*}$ has order at most 2, then $(\alpha E, \theta \alpha E)$ is a t.i. $2 m$-space that meets each member of $\Sigma_{\star}$ in an $m$-space.

Finally, if there are two subspaces $(E, \theta E)$ and $(\alpha E, \theta \alpha E)$, then $\alpha \notin E$ and these have intersection 0 .

Proof of Theorem 3.1. Let $\Sigma$ and $\Sigma_{\star}$ be as above. By the lemma, there are t.i. $2 m$-spaces $Z$ (if $q$ is even) or $Z, Z^{\prime}$ (if $q$ is odd) such that $\Sigma_{\star}$ is the set of elements 
of $\Sigma$ met nontrivially by either of these $2 m$-spaces. Then

$$
\Sigma^{\bullet}:= \begin{cases}\left(\Sigma-\Sigma_{\star}\right) \cup\{Z\} & \text { if } q \text { is even } \\ \left(\Sigma-\Sigma_{\star}\right) \cup\left\{Z, Z^{\prime}\right\} & \text { if } q \text { is odd }\end{cases}
$$

is a symplectic partial spread of size $q^{2 m}-q^{m}+(2, q-1)$.

Maximality: Suppose that $X$ is a t.i. $2 m$-space meeting each member of $\Sigma^{\bullet}$ in zero. Since $\Sigma$ is a spread, the set $\Sigma_{X}$ of members of $\Sigma$ meeting $X$ nontrivially must be contained in $\Sigma_{\star}$. If $(\star) \Sigma_{X}=\Sigma_{\star}$ and $\operatorname{dim} X \cap Y=0$ or $m$ for each $Y \in \Sigma$, then $X=Z$ or $Z^{\prime}$ by Lemma 3.2, which contradicts the fact that $X \notin \Sigma^{\bullet}$.

We count in order to prove $(\star)$. Let $a_{i}$ be the number of $Y \in \Sigma$ such that $\operatorname{dim} X \cap Y=i$, where $1 \leq i \leq 2 m-1$. Since the intersections $X \cap Y$ produce a partition of $X-\{0\}$,

$$
\begin{aligned}
\sum_{1}^{2 m-1} a_{i}\left(q^{i}-1\right) & =q^{2 m}-1 \\
\sum_{1}^{2 m-1} a_{i} & =\left|\Sigma_{X}\right| \leq\left|\Sigma_{\star}\right|=q^{m}+1 .
\end{aligned}
$$

There cannot be two subspaces of $X$ of dimension $>m$ and $\geq m$ having zero intersection. Thus, if $a_{k} \neq 0$ for some $k>m$ then $a_{k}=1$ and $a_{i}=0$ whenever $m \leq i \leq 2 m-1, i \neq k$. This produces the contradiction $q^{2 m}-1=\left(q^{k}-1\right)+$ $\sum_{1}^{m-1} a_{i}\left(q^{i}-1\right) \leq\left(q^{k}-1\right)+\sum_{1}^{m-1} a_{i}\left(q^{m-1}-1\right) \leq\left(q^{k}-1\right)+\left(q^{m}+1-1\right)\left(q^{m-1}-1\right)$.

Thus, $a_{k}=0$ for $k>m$, and $q^{2 m}-1=\sum_{1}^{m} a_{i}\left(q^{i}-1\right) \leq \sum_{1}^{m} a_{i}\left(q^{m}-1\right) \leq$ $\left(q^{m}+1\right)\left(q^{m}-1\right)$. Then $a_{i}=0$ for $i<m$ and $a_{m}=q^{m}+1$, as required.

Remarks 3.3. When $2 m=4$ the theorem is a special case of CDFS, ThK and Theorem 9.1 which suggests the question: Can more than one subset like $\Sigma_{\star}$ be removed in Theorem 3.1]

The last part of the proof showed that a partition of the nonzero vectors of $\mathbb{F}_{q}^{2 m}$ induced by a set of proper subspaces has size at least $q^{m}+1$, with equality if and only if the subspaces all have dimension $m$.

\section{Orthogonal spreads}

Let $V$ be an $\mathrm{O}^{+}(4 m, q)$-space (for even $q$ and $4 m \geq 8$ ) with quadratic form $Q$. Then $V$ has an orthogonal spread $\Sigma$, and $|\Sigma|=q^{2 m-1}+1$ (first proved in [Dil], then rediscovered in $\mathrm{Dye}$; cf. [Ka2, $\mathrm{KaW}]$ ). This leads to our simplest examples:

Proposition 4.1. $\Sigma$ is a maximal partial spread of size $q^{2 m-1}+1$, and is symplectic.

Proof. For even $q$, t.s. subspaces are also t.i., so $\Sigma$ is symplectic. Maximality: since $2 m>2$, the quadratic form induced by $Q$ on any $2 m$-space has a nontrivial zero. Thus, every $2 m$-space has nonzero intersection with some member of $\Sigma$.

Remark 4.2. If $d=2^{2 m}$ and $q=2$ then $|\Sigma|=\frac{1}{2} d+1$. Finding maximal symplectic partial spreads of size $\frac{1}{2} d+1$ appears to be a goal of [MBGW].

Lemma 4.3. Let $E=\mathbb{F}_{q} \subseteq F=\mathbb{F}_{q^{k}}$ with $q$ even. If $X$ is an E-subspace of an orthogonal $F$-space and $|X|>q^{k^{2}+k}$, then $X$ contains a nonzero $F$-singular vector.

Proof. We are given an $F$-space $V$ equipped with a quadratic form $Q$ and associated bilinear form $f($,$) ; both forms have values in F$ not in $E$. The symbol $\perp$ will refer to the $F$-space $V$, while \langle\rangle$_{L}$ refers to spanning an $L$-subspace for $L=E$ or $F$. 
For $i=1, \ldots, k+1$, we will construct $E$-linearly independent vectors $x_{1}, \ldots, x_{i} \in$ $X$ and an $E$-subspace $X_{i}$ such that $\left\langle x_{1}, \ldots, x_{i}\right\rangle_{E} \leq X_{i} \leq\left\langle x_{1}, \ldots, x_{i}\right\rangle_{F}^{\perp} \cap X$ and $\left|X_{i}\right| \geq|X| /|F|^{i}$. (In particular, $x_{1}, \ldots, x_{k+1} \in\left\langle x_{1}, \ldots, x_{k+1}\right\rangle_{F}^{\perp} \cap X$.)

Let $0 \neq x_{1} \in X$ and $X_{1}:=\left\langle x_{1}\right\rangle_{F}^{\perp} \cap X$. Then $x_{1} \in X_{1}$ (since $q$ is even and hence $V$ is symplectic) and $\left|X_{1}\right|=\left|\left\langle x_{1}\right\rangle_{F}^{\perp}\right||X| /\left|\left\langle x_{1}\right\rangle_{F}^{\perp}+X\right| \geq\left|\left\langle x_{1}\right\rangle_{F}^{\perp}\right||X| /|V|=|X| /|F|$.

For induction, let $1 \leq i \leq k$ and assume that we have $x_{1}, \ldots, x_{i}$ and $X_{i}$. Then $\left|X_{i}\right| \geq|X| /|F|^{i}>q^{k^{2}+\bar{k}} /\left(q^{\bar{k}}\right)^{k} \geq\left|\left\langle x_{1}, \ldots, x_{i}\right\rangle_{E}\right|$. Let $x_{i+1} \in X_{i}-\left\langle x_{1}, \ldots, x_{i}\right\rangle_{E}$ and $X_{i+1}:=\left\langle x_{i+1}\right\rangle_{F}^{\perp} \cap X_{i}$. Then $x_{i+1} \in X_{i+1} \leq\left\langle x_{i+1}\right\rangle_{F}^{\perp} \cap\left\langle x_{1}, \ldots, x_{i}\right\rangle_{F}^{\perp} \cap X$ and $\left|X_{i+1}\right|=\left|\left\langle x_{i+1}\right\rangle_{F}^{\perp}\right|\left|X_{i}\right| /\left|\left\langle x_{i+1}\right\rangle_{F}^{\perp}+X\right| \geq\left(|X| /|F|^{i}\right) /|F|$, as needed for induction.

Since $\left\langle x_{1}, \ldots, x_{k+1}\right\rangle_{E}$ is in $X \cap\left\langle x_{1}, \ldots, x_{k+1}\right\rangle_{E}^{\perp}$ and has size $q^{k+1}>|F|$, the additive map $Q:\left\langle x_{1}, \ldots, x_{k+1}\right\rangle_{E} \rightarrow F$ has a nonzero kernel.

Remarks 4.4. The preceding argument did not require anything about the nature of the quadratic form, which could even have a large radical.

Although the argument used the fact that all vectors are isotropic, it can still be used for unitary spaces and orthogonal spaces of odd characteristic. One minor difference is that we need to know that $X_{i}$ has an isotropic vector $x_{i+1} \in X_{i}-$ $\left\langle x_{1}, \ldots, x_{i}\right\rangle_{E}$. This is clear if $X_{i}$ is the span of its isotropic vectors; and that holds unless $X_{i} / \operatorname{rad} X_{i}$ is anisotropic, hence of dimension 1 or (in the orthogonal case) 2 . Thus, there is a choice $x_{i+1}$ for each $i$ if we replace the condition $|X|>q^{k^{2}+k}$ by $|X|>\left(q^{2}\right)^{k^{2}+k+1}$ for unitary spaces and by $|X|>q^{k^{2}+k+2}$ for orthogonal spaces.

These observations do not, however, lead to useful unitary or odd characteristic orthogonal analogues of the next theorem: unfortunately, there is no unitary spread in dimension $\geq 6$ [Th] and no known odd characteristic orthogonal spread in dimension $>8$.

Theorem 4.5. If $q$ is even and $m>(k+1) / 2$, then $\mathbb{F}_{q}^{4 m k}$ has a maximal partial spread of size $q^{2 m k-k}+1$ that is orthogonal and hence also symplectic.

Proof. Let $V$ be an $\mathrm{O}^{+}\left(4 m, q^{k}\right)$-space with quadratic form $Q$, and let $\Sigma$ be an orthogonal spread in $V$. Let $T: \mathbb{F}_{q^{k}} \rightarrow \mathbb{F}_{q}$ be the trace map. Then $Q^{\prime}(v):=T(Q(v))$ is a quadratic form that turns $V$ into an $\mathrm{O}^{+}(4 m k, q)$-space. Moreover, $\Sigma$ is still an orthogonal partial spread in this space, of size $\left(q^{k}\right)^{2 m-1}+1$.

Maximality: If $X$ is an $\mathbb{F}_{q}$-subspace of $V$ of dimension $2 m k$, then $|X|=q^{2 m k}>$ $q^{k^{2}+k}$. By Lemma 4.3. $X$ contains a nonzero $\mathbb{F}_{q^{k}}$-singular vector that must lie in some member of the $\mathrm{O}^{+}\left(4 m, q^{k}\right)$-spread $\Sigma$. Thus, $X$ has nonzero intersection with a member of $\Sigma$.

Question 4.6. Is every $\mathrm{O}^{+}\left(4 m, q^{k}\right)$-spread also a maximal orthogonal partial spread in an $\mathrm{O}^{+}(4 m k, q)$-space? This seems plausible since it is correct when either $m>(k+1) / 2$ (Theorem 4.5) or $m=2$ Gr] (cf. Theorem 5.2(i)).

Remarks 4.7. If $q=2$ and $d=2^{2 m k}$ with $m>(k+1) / 2$, then the maximal symplectic partial spreads in Theorem 4.5 have size $\frac{1}{2^{k}} d+1$, resembling Remark 4.2. Grassl's computer data Gr] suggests much more: for even $q$ there appears to be a maximal symplectic partial spread of size $2^{i}+1$ in $\operatorname{Sp}(2 n, q)$-space whenever $q^{n / 2}+1 \leq 2^{i}+1 \leq q^{n}+1$.

We will need the following elementary observation several times: 
Lemma 4.8. If $\Sigma$ is a maximal orthogonal partial spread of an $\mathrm{O}^{+}(4 m, q)$-space with $q$ even and $m \geq 2$, then it is also a maximal symplectic partial spread.

Proof. Suppose that $Y \notin \Sigma$ is a t.i. $2 m$-space such that $\Sigma \cup\{Y\}$ is a symplectic partial spread. The quadratic form on $V$ restricts to a semilinear map on the t.i. subspace $Y$; the kernel is a t.s subspace $Y_{0}$ of dimension $\geq 2 m-1$. If $\operatorname{dim} Y_{0}=2 m$ then $Y=Y_{0}$ must have the same type as the members of $\Sigma$ (cf. Section 2).

In any case let $W$ be the t.s. $2 m$-space containing $Y_{0}$ having the same type as the members of $\Sigma$. By maximality, $\Sigma \cup\{W\}$ is not an orthogonal partial spread, so that $W \cap X \neq 0$ for some $X \in \Sigma$. Since $\operatorname{dim} W \cap X \equiv 2 m(\bmod 2)$ we have $\operatorname{dim} W \cap X \geq 2$. Since $Y_{0}, W \cap X \leq W$ and $\operatorname{dim} Y_{0} \geq 2 m-1$, it follows that $Y_{0} \cap(W \cap X) \neq 0$ and hence that $Y \cap X \neq 0$. This contradicts the fact that $\Sigma \cup\{Y\}$ is a partial spread.

$$
\text { 5. } \mathrm{O}^{+}\left(4, q^{k}\right) \text {-SPACE }
$$

Example 5.1. If $q$ is even then an $\operatorname{Sp}(4, q)$-space has a maximal symplectic partial spread of size $q+1$ that is also a maximal orthogonal partial spread.

Proof. An $\mathrm{O}^{+}(4, q)$-space has $(q+1)^{2}$ singular points partitioned by exactly two orthogonal spreads $\Sigma, \Sigma^{\dagger}$, arising from the two types of t.s. 2-spaces (cf. Section 2); each member of $\Sigma$ and each member of $\Sigma^{\dagger}$ meet nontrivially. Possibly the most elementary (and most opaque) way to see that these are maximal symplectic spreads is to count the number of t.i. lines containing at least one singular point. There are $2|\Sigma|+(q+1)^{2}(q-1)=\left(q^{2}+1\right)(q+1)$ such lines, which is exactly the total number of t.i. lines.

Theorem 5.2. Let $q$ be even and $k \geq 1$.

(i) An $\mathrm{Sp}(4 k, q)$-space has a maximal symplectic partial spread of size $q^{k}+1$ that is also a maximal orthogonal partial spread.

(ii) An $\mathrm{Sp}(4 k, q)$-space has a maximal symplectic partial spread of size $2 q^{k}+1$.

Proof. (i) The preceding example produces a maximal symplectic partial spread $\Sigma$ of an $\operatorname{Sp}\left(4, q^{k}\right)$-space $V$ that is also a maximal orthogonal partial spread. Viewed over $\mathbb{F}_{q}$ (using a trace map as in the proof of Theorem 4.5) the set $\Sigma$ again is an orthogonal partial spread. It is a maximal symplectic partial spread by $\mathbf{G r}$, and hence also a maximal orthogonal partial spread.

We include slightly more detail: in $\overline{\mathrm{Gr}}$ the $\mathbb{F}_{q^{-} \text {-space }}\left(\mathbb{F}_{q^{k}}^{2}\right)^{2}$ is equipped with the alternating bilinear form $\left((u, v),\left(u^{\prime}, v^{\prime}\right)\right):=T\left(u \cdot v^{\prime}-u^{\prime} \cdot v\right)$ using the trace map $T: \mathbb{F}_{q^{k}} \rightarrow \mathbb{F}_{q}$. The partial spread $\Sigma$ consists of the t.i. subspaces $\left\{\left(0,0, y_{1}, y_{2}\right) \mid\right.$ $\left.y_{1}, y_{2} \in \mathbb{F}_{q^{k}}\right\}$ and $\left\{\left(x_{1}, x_{2}, x_{2} \alpha, x_{1} \alpha\right) \mid x_{1}, x_{2} \in \mathbb{F}_{q^{k}}\right\}$ for each $\alpha \in \mathbb{F}_{q^{k}}$. These are t.s. $2 k$-spaces for the quadratic form $Q(u, v)=T(u \cdot v)$. In the preceding example, $\Sigma^{\dagger}$ is $\Sigma^{j}$, where $j:\left(x_{1}, x_{2}, y_{1}, y_{2}\right) \mapsto\left(x_{1}, y_{1}, x_{2}, y_{2}\right)$.

(ii) Choose any $Z \in \Sigma$. Obtain a new symplectic partial spread $\Sigma \bullet$ by removing $Z$ and then, for each 1-dimensional $\mathbb{F}_{q^{k}}$-subspace $W$ of $Z$, adjoining one 2-dimensional t.i. $\mathbb{F}_{q^{k}}$-subspace that contains $W$ and is different from both $Z$ and the member of $\Sigma^{\dagger}$ containing $W$. This produces a maximal symplectic partial spread of the

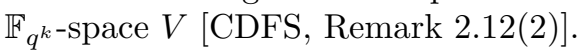


In fact $\Sigma \bullet$ is also a maximal symplectic partial spread of the $\mathbb{F}_{q}$-space $V$. For, let $X$ be a t.i. $2 k$-dimensional $\mathbb{F}_{q}$-subspace of $V$ having zero intersection with all members of $\Sigma^{\bullet}$. By (i), $X$ has nonzero intersection with some member of $\Sigma$, which therefore must be $Z$. Then $X$ has nonzero intersection with some $\mathbb{F}_{q^{k}}$-point $W$ of $Z$ and hence with the adjoined $\mathbb{F}_{q^{k}}$-space in $\Sigma^{\bullet}$ containing $W$, which is a contradiction.

Part (i) contains Theorem 7.7(i) as a special case, and amounts to the case $m=1$ not dealt with in Theorem 4.5

The proof of (i) in $\mathrm{Gr}$ uses a neat computational idea. Unsupported optimism suggests that there should also be a nice geometric proof.

Example 5.3. Proposition 5.2(i) points to a general construction (compare Remarks (7.8). Let $V=\mathbb{F}_{q}^{4 m}$ be an orthogonal, symplectic or unitary space. Let $X$ and $Y$ be t.i./t.s. $2 m$-spaces with zero intersection, and let $\Sigma_{X}$ be a partial spread (of $m$-spaces) of $X$. Each $A \in \Sigma_{X}$ determines another $m$-space $A^{\prime}:=A^{\perp} \cap Y$, and $A+A^{\prime}$ is a t.i./t.s. $2 m$-space. Then $\Sigma:=\left\{A+A^{\prime} \mid A \in \Sigma_{X}\right\}$ is a partial spread of the same type as the underlying space $V$. (If $A \neq B \in \Sigma_{X}$ then $V=X \oplus Y=(A \oplus B) \oplus\left(A^{\prime} \oplus B^{\prime}\right)$, so that $A \oplus A^{\prime}$ and $B \oplus B^{\prime}$ have zero intersection.)

When $\Sigma_{X}$ is a maximal partial spread (or even a spread), some of these partial spreads may be maximal orthogonal, symplectic or unitary partial spreads of size $q^{m}+1$ (as in Proposition 5.2(i) and Theorem 7.7), but we do not see how to prove that. (See Question 7.6 for instances of such symplectic partial spreads that are not maximal. As noted earlier, there is no unitary spread in dimension $\geq 6$ Th.

\section{Projections}

Let $q$ be even. A key ingredient of $[\mathrm{Ka2}, \mathrm{Ka} 3, \underline{\mathrm{KaW}}$ is the fact that there is a natural transition between $\mathrm{O}^{+}(4 m, q)$-spreads and $\mathrm{Sp}(4 m-2, q)$-spreads. This uses any nonsingular point $z$ of an $\mathrm{O}^{+}(4 m, q)$-space and projects into the symplectic space $z^{\perp} / z$. This procedure also applies to orthogonal and symplectic partial spreads:

Lemma 6.1. Let $z$ be a nonsingular point of an $\mathrm{O}^{+}(4 m, q)$-space $V$.

(i) If $\Sigma$ is a maximal orthogonal partial spread of $V$, then $\Sigma / z:=$ $\left\{\left\langle z^{\perp} \cap X, z\right\rangle / z \mid X \in \Sigma\right\}$ is a maximal symplectic partial spread of the $\operatorname{Sp}(4 m-2, q)$-space $z^{\perp} / z$.

(ii) If $\Sigma^{\prime}$ is a maximal symplectic partial spread of $z^{\perp} / z$, then there is a maximal orthogonal partial spread $\Sigma$ of $V$ such that $\Sigma^{\prime}=\Sigma / z$. Moreover, $\Sigma$ is a maximal symplectic partial spread.

(iii) If $\Sigma_{1}$ is a maximal orthogonal partial spread of $V$ and $z_{1}$ is a nonsingular point of $V$, then $\Sigma / z$ and $\Sigma_{1} / z_{1}$ are equivalent symplectic partial spreads if and only if $\Sigma_{1}$ is the image of $\Sigma$ under an automorphism of the orthogonal geometry of $V$ that sends $z$ to $z_{1}$.

Proof. (i) $\Sigma / z$ is a symplectic partial spread: If $X$ and $Y$ are distinct members of $\Sigma$ and $\left\langle z^{\perp} \cap X, z\right\rangle \cap\left\langle z^{\perp} \cap Y, z\right\rangle \neq z$, then $z \in\langle x, y\rangle$ for some points $x \in z^{\perp} \cap X$, $y \in z^{\perp} \cap Y$. Then $x$ and $y$ are perpendicular to $z$ and hence to one another, so that $\langle x, y\rangle$ is t.s. whereas $z$ is nonsingular.

Maximality: Suppose that $(\Sigma / z) \cup\{U / z\}$ is a larger symplectic partial spread for a t.i. $2 m$-space $U$ of $V$ containing $z$. Let $U^{\prime}$ be the hyperplane of $U$ consisting of singular vectors (i.e., $U^{\prime}$ is the kernel of the semilinear map $U \rightarrow \mathbb{F}_{q}$ induced by the 
quadratic form on $V$ ). The members of $\Sigma$ all have the same type (cf. Section 2). Let $\hat{U}$ be the t.s. $2 m$-space of that type containing $U^{\prime}$. Then $\hat{U}$ meets each $X \in \Sigma$ in at most a 1 -space and hence only in 0 (by Section $21 \geq \operatorname{dim}(\hat{U} \cap X) \equiv 2 m$ $(\bmod 2)$ and hence $\hat{U} \cap X=0)$. Thus, $\Sigma \cup\{\hat{U}\}$ is an orthogonal partial spread properly containing $\Sigma$, whereas $\Sigma$ is assumed to be maximal.

(ii) Choose a type of t.s. $2 m$-space of $V$. If $U / z \in \Sigma^{\prime}$ let $U^{\prime}$ be the hyperplane of singular vectors of the t.i. $2 m$-space $U$, and let $\hat{U}$ be the t.s. $2 m$-space containing $U^{\prime}$ of the chosen type. Then the set $\Sigma$ consisting of these subspaces $\hat{U}$ is an orthogonal partial spread: distinct members of $\Sigma$ meet in at most a 1 -space and hence have intersection 0 since members of $\Sigma$ have the same type. Clearly $\Sigma^{\prime}=\Sigma / z$.

Maximality: If $\Sigma^{+}$is an orthogonal partial spread properly containing $\Sigma$, then $\Sigma^{+} / z$ properly contains $\Sigma / z=\Sigma^{\prime}$, whereas $\Sigma^{\prime}$ is maximal.

The final statement follows from Lemma 4.8 .

(iii) As a consequence of Witt's Lemma [Ta, p. 57], an equivalence from $\Sigma / z$ to $\Sigma_{1} / z_{1}$ lifts first to $z^{\perp} \rightarrow z_{1}^{\perp}$ and then to an automorphism of the orthogonal geometry on $V$ sending $z \rightarrow z_{1}$ and $\Sigma \rightarrow \Sigma_{1}$. The converse is clear.

By (iii), a maximal orthogonal partial spread $\Sigma$ produces many inequivalent maximal symplectic partial spreads for different choices of $z$, where the number of inequivalent ones requires knowledge of the automorphism group of $\Sigma$. This was crucial in [Ka2, Ka3, KaW].

Theorem 6.2. If $k \geq 2$ then there is a maximal partial $\mathrm{Sp}(4 k-2, q)$-spread of size $q^{k}+1$.

Proof. Use Lemma 6.1(i) and Theorem 5.2(i).

Theorem 6.3. If $m>(k+1) / 2$ then there is a maximal partial $\operatorname{Sp}(4 m k-2, q)$ spread of size $q^{2 m k-k}+1$.

Proof. Use Lemma 6.1(i) and Theorem 4.5 .

\section{8-DIMENSIONAL PARTIAL SPREADS}

In $\mathrm{O}^{+}(8, q)$-spaces, triality [Ti1] allows us to use more easily visualized points and partial ovoids in place of partial spreads: a triality map sends orthogonal (partial) ovoids to orthogonal (partial) spreads. This produces maximal partial $\operatorname{Sp}(8, q)$-spreads when $q$ is even.

7.1. 8-dimensional ovoids. Spreads and ovoids are known in $\mathrm{O}^{+}(8, q)$-spaces when $q$ is prime, a power of 2 or 3 , or $\equiv 2(\bmod 3)$ (some of these ovoids are described in [Ka1]). They have size $q^{3}+1$.

Lemma 7.1. Let $\Omega$ be an ovoid in an $\mathrm{O}^{+}(8, q)$-space $V$, where $q>2$. Let $a \notin \Omega$ be a singular point that is the only singular point in $\left\langle a^{\perp} \cap \Omega\right\rangle^{\perp}$. (Examples appear below in Appendix $\mathrm{A}$ for all even $q>2$.) Then $\Omega^{\bullet}:=\left(\Omega-\left(a^{\perp} \cap \Omega\right)\right) \cup\{a\}$ is a maximal orthogonal partial ovoid of size $q^{3}-q^{2}+1$.

Proof. Clearly $\Omega^{\bullet}$ is an orthogonal partial ovoid. If $b$ is a singular point not perpendicular to any member of $\Omega^{\bullet}$ then $b^{\perp} \cap \Omega \subseteq a^{\perp} \cap \Omega$. Since both of these sets have size $q^{2}+1$ (e.g., by [Ka1, p. 1196]), we obtain the contradiction that both $a$ and $b$ are the singular point in $\left\langle a^{\perp} \cap \Omega\right\rangle^{\perp}$. 
Theorem 7.2. Applying triality $\tau$ to the preceding lemma produces a maximal orthogonal partial spread $\Sigma^{\bullet}:=\Omega^{\bullet} \tau$ of size $q^{3}-q^{2}+1$ in an $\mathrm{O}^{+}(8, q)$-space when $q>2$. If $q$ is even then $\Sigma^{\bullet}$ is a maximal symplectic partial spread.

In particular, such maximal symplectic partial spreads exist for all even $q$.

Proof. By the previous lemma, $\Sigma^{\bullet}$ is a maximal orthogonal partial spread. If $q$ is even use Lemma4.8.

We can imitate the preceding result and remove several sets $a^{\perp} \cap \Omega$ by using a specific type of ovoid.

Theorem 7.3. If $q>2$ is even and $1 \leq s \leq q / 5$, then an $\mathrm{O}^{+}(8, q)$-space has maximal orthogonal partial spreads of size $n_{s}=q^{3}-s q^{2}+(s-1)(q+2)+\left(\begin{array}{c}s \\ 2\end{array}\right)(q-2)+1$. There is also a maximal orthogonal partial spread of size $n_{4}-1$ if $q \geq 16$.

These are also maximal symplectic partial spreads.

Proof. As in Theorem 7.2 we will construct maximal orthogonal ovoids. Since this the only part of this paper involving detailed computations, those computations have been postponed to Appendix $\mathrm{A}$

For the ovoid $\Omega$ in Appendix $\mathrm{A}$ Example A.13(i) provides us with many sets $\mathcal{P}$ of $s$ singular points disjoint from $\Omega$ together with the sizes $\left|\bigcap_{p \in \mathcal{P}^{\prime}} p^{\perp} \cap \Omega\right|$ for all $\mathcal{P}^{\prime} \subseteq \mathcal{P}$. Then

$$
\Omega_{s}^{\bullet}:=\left(\Omega-\bigcup_{p \in \mathcal{P}}\left(p^{\perp} \cap \Omega\right)\right) \cup \mathcal{P}
$$

is an orthogonal partial ovoid of size $\left(q^{3}+1\right)-s\left(q^{2}+1\right)+\left(\begin{array}{c}s \\ 2\end{array}\right)(2 q)-\left(\begin{array}{c}s \\ 3\end{array}\right)(q+2)+$ $\cdots \pm\left(\begin{array}{l}s \\ s\end{array}\right)(q+2)+s=\left(q^{3}+1\right)-s\left(q^{2}+1\right)+\left(\begin{array}{c}s \\ 2\end{array}\right)(2 q)-(q+2)+s(q+2)-\left(\begin{array}{l}s \\ 2\end{array}\right)(q+$ $2)+(1-1)^{s}(q+2)+s$.

Maximality of $\Omega_{s}^{\bullet}$ : Suppose that $b$ is a singular point not perpendicular to every member of $\Omega_{s}^{\bullet}$. Since $\Omega$ is an orthogonal ovoid, $b^{\perp} \cap \Omega$ must be contained in $\bigcup_{p \in \mathcal{P}}\left(p^{\perp} \cap \Omega\right)$. By Lemma A.2, $s(5 q-5) \geq \sum_{p \in \mathcal{P}}\left|b^{\perp} \cap p^{\perp} \cap \Omega\right| \geq\left|b^{\perp} \cap \Omega\right|=q^{2}+1$, which contradicts our assumption that $s \leq q / 5$.

The same argument can be used for Example A.13(ii), producing the stated additional maximal orthogonal partial spreads.

Use Lemma 4.8 for the final assertion.

The preceding proof should be compared to the proofs of Theorem 7.14 and the more elementary Theorem 9.1. In those proofs the needed intersection sizes are known for simple geometric reasons. Here there does not seem to be a geometric explanation for the various intersection sizes occurring in Appendix A.

7.2. 4- and 5-dimensional orthogonal ovoids. The next 8-dimensional partial spreads (in Theorem 7.7) arise from small-dimensional ovoids.

Example 7.4. If $\Omega$ is an $\mathrm{O}^{-}(4, q)$-ovoid (i. e., an elliptic quadric) in an $\mathrm{O}^{-}(4, q)$ space $W$ inside a nondegenerate orthogonal $\mathbb{F}_{q}$-space $V$, then $\Omega$ is a maximal orthogonal partial ovoid of $V$.

Proof. If $x$ is any point of $V$ then $x^{\perp} \cap W$ contains a hyperplane of $W$ and hence contains either $p^{\perp} \cap W$ for a singular point $p$ of $W$ or $n^{\perp} \cap W$ a nonsingular point $n$ of $W$. Each such hyperplane of $W$ contains a singular point of $W$, and hence meets $\Omega$ nontrivially.

A more general version of this example is a simple consequence of 5-dimensional results BS1, Ba, (also see Lemma B.1): 
Lemma 7.5. If $\Omega$ is an ovoid in an $\mathrm{O}(5, q)$-subspace of a nondegenerate orthogonal $\mathbb{F}_{q}$-space $V$, then it is a maximal orthogonal partial ovoid of $V$.

Proof. Once again we will show that each point $x$ of $V$ is perpendicular to some point in $\Omega$. We may assume that $U:=\langle\Omega\rangle \not{Z} x^{\perp}$, so that $H:=x^{\perp} \cap U$ is a hyperplane of $U$. By the preceding example, we may also assume that $U$ is not of type $\mathrm{O}^{-}(4, q)$.

If $H$ has type $\mathrm{O}^{+}(4, q)$ then $H$ contains a t.s. line, and each t.s. line of $U$ meets each ovoid of $U$ (by definition; see Section 2).

If $H$ has type $\mathrm{O}^{-}(4, q)$ then its set $\Lambda$ of singular points is a classical quadric. Then $\Lambda \cap \Omega \neq \emptyset[\mathrm{BS} 1, \mathrm{Ba}]$.

Thus, $H$ is degenerate. If there is a singular point $y$ in its radical $\operatorname{rad} H$, then every t.s. line of $U$ on $y$ meets $\Omega$ at a point perpendicular to $y$.

Finally, if $\operatorname{rad} H$ is a nonsingular point then $q$ is even and the radical $r$ of $U$ is in $H$ (since all hyperplanes of $U$ not containing its radical are nonsingular). Let "bar" denote the projection map $U \rightarrow U / r$. Then $\bar{H}$ is a tangent or secant plane of the ovoid $\bar{\Omega}$ in the 4 -space $\bar{U}$, so that $\bar{H}$ contains 1 or $q+1$ points of $\bar{\Omega}$. If $T / r$ is one of these points, then the line $T$ has a unique singular point, and this lies in both $H \leq x^{\perp}$ and $\Omega$.

Question 7.6. Which ovoids in orthogonal spaces are partial ovoids in all largerdimensional orthogonal spaces over the same field? This requires that all hyperplanes of the smaller orthogonal space meet the ovoid. Perhaps this does not hold for any ovoids of $\mathrm{O}^{+}(6, q)$-spaces that span the underlying 6-space (and there are, indeed, many such ovoids for which this requirement does not hold). However, this requirement does hold for some of the known $\mathrm{O}^{+}(8, q)$ ovoids, as in Co, Theorem 3.9] and for the ovoids in [Ka1, Sec. 7] and Appendix \$,

Theorem 7.7. Let q be a prime power.

(i) There are inequivalent maximal partial $\mathrm{O}^{+}(8, q)$-spreads $\Sigma$ of size $q^{2}+1$ :

(a) One for which $\operatorname{Sp}(8, q)_{\Sigma}$ has a subgroup $\mathrm{SL}\left(2, q^{2}\right)$ acting 2-transitively on $\Sigma$; and

(b) One occurring when $q$ is odd but not prime and for which $\operatorname{Sp}(8, q)_{\Sigma}$ is intransitive on $\Sigma$.

(ii) If $q=2^{e}$ then there are inequivalent maximal partial $\operatorname{Sp}(8, q)$-spreads $\Sigma$ of size $q^{2}+1$ that are orthogonal partial spreads:

(a) One for which $\mathrm{Sp}(8, q)_{\Sigma}$ has a subgroup $\mathrm{SL}\left(2, q^{2}\right)$ acting 2-transitively on $\Sigma$; and

(b) One occurring when $e>1$ is odd and for which $\operatorname{Sp}(8, q)_{\Sigma}$ has a subgroup $\mathrm{Sz}(q)$ acting 2-transitively on $\Sigma$.

Proof. Let $\tau$ be a triality map for an $\mathrm{O}^{+}(8, q)$-space $V$. For $\Omega$ in the preceding example or lemma, $\Sigma=\Omega^{\tau}$ is a maximal orthogonal partial spread of $V$.

For (ia) use an elliptic quadric, whose group of isometries produces the last part. For (ib) there are other choices for $\Omega$ in Lemma 7.5, such as those in [Ka1, Sec. 5].

If $q$ is even then Lemma 7.5 applies, where the only known choices for $\Omega$ are an elliptic quadric (Example 7.4) and a Suzuki-Tits ovoid (see Appendix B). The stated groups arise from subgroups of $\Omega^{+}(8, q)$ acting on $\Omega$.

The various partial spreads are inequivalent as orthogonal partial spreads, since the corresponding maximal orthogonal partial ovoids $\Sigma^{\tau^{-1}}=\Omega$ are inequivalent. 
However, this is orthogonal inequivalence, which is not the same as symplectic inequivalence in (ii).

Nevertheless, the symplectic partial spreads in (iia) and (iib) are inequivalent. This can be proved using the groups appearing in (iia) and (iib), but a geometric proof is simpler. The construction of the orthogonal partial spread in (iia) provides us with $q^{2}+1$ t.i. 4 -spaces (in fact, t.s. 4 -spaces in $\Omega^{\perp \tau}$ ) meeting each of its members. If the symplectic partial spreads are equivalent then (iib) has $q^{2}+1$ t.i. 4 -spaces $U$ meeting each of its members. Then $U$ is t.s. since it is spanned by singular vectors. Now $(\Sigma \cup\{U\})^{\tau^{-1}}=\Omega \cup\left\{U^{\tau^{-1}}\right\}$ for a singular point $U^{\tau^{-1}}$ of $\Omega^{\perp}$. This contradicts the fact that, for (iib), the 3 -space $\Omega^{\perp}$ contains only $q+1$ singular points.

Remarks 7.8. We excluded $q=2$ in (iib) since that produces the same partial spread as in (iia). Part (iia) is a very special case of a result in Gr. (cf. Theorem 5.2). Is there an analogous generalization of (ii)?

Note that $\operatorname{Sp}(8, q)_{\Sigma}$ even contains subgroups $\operatorname{SL}\left(2, q^{2}\right) \times \operatorname{SL}\left(2, q^{2}\right)$ in (i) and $\mathrm{Sz}(q) \times \mathrm{O}(3, q) \cong \mathrm{Sz}(q) \times \mathrm{SL}(2, q)$ in (iib).

In both (i) and (ii) there are t.s. 4-spaces $X, Y$ such that the members of $\Sigma$ meet $X$ and $Y$ in spreads of each (cf. Example 5.3).

See $[\mathrm{PW}]$ for a survey of $\mathrm{O}(5, q)$-ovoids.

7.3. Extending a partial spread. How can one search for maximal symplectic partial spreads? One obvious answer is to start with a symplectic or orthogonal partial spread and try to extend it to a maximal one (this was the computational method used to produce the table in $[\mathrm{Gr}$ ). The instances considered below may have extensions to maximal ones other than the ones we provide.

Once again, points are easier to deal with than subspaces.

7.3.1. $\mathrm{O}^{-}(4, q)$-ovoids. A simple example of an orthogonal partial ovoid is ( $\Omega-$ $\{p\}) \cup\{x\}$, where $p$ is a point in the set $\Omega$ of singular points of an $\mathrm{O}^{-}(4, q)$-space $U$ and $x \notin U$ is a singular point in $\left(p^{\perp} \cap U\right)^{\perp}-U^{\perp}$.

Lemma 7.9. For any $q$ an $\mathrm{O}^{+}(8, q)$-space has a maximal orthogonal partial ovoid of size $2 q^{2}+1$.

Proof. In an $\mathrm{O}^{+}(8, q)$-space $V$ consider anisotropic 2-spaces $A, A^{\prime}$ and a totally singular 2-space $\left\langle p, p^{\prime}\right\rangle$ such that $\left\langle A, A^{\prime}, p, p^{\prime}\right\rangle=A \perp A^{\prime} \perp\left\langle p, p^{\prime}\right\rangle$. Let $E=\langle A, p\rangle$ and $E^{\prime}=\left\langle A^{\prime}, p^{\prime}\right\rangle$, and let $x$ be a point of $\left\langle p, p^{\prime}\right\rangle-\left\{p, p^{\prime}\right\}$.

Let $U$ and $U^{\prime}$ be non-perpendicular $\mathrm{O}^{-}(4, q)$-subspaces of $V$ such that $E^{\prime \perp}>$ $U>E$ and $E^{\perp}>U^{\prime}>E^{\prime}$. (In order to construct these, note that $p$ and $p^{\prime}$ are in t.s. lines $\neq\left\langle p, p^{\prime}\right\rangle$ of the $\mathrm{O}^{+}(4, q)$-space $\left(A \perp A^{\prime}\right)^{\perp}$. Choose singular points $u, u^{\prime} \in\left(A \perp A^{\prime}\right)^{\perp}-\left\langle p, p^{\prime}\right\rangle$ perpendicular to $p^{\prime}$ and $p$, respectively, but not to each other. Then $U:=A \perp\langle p, u\rangle=\langle E, u\rangle$ and $U^{\prime}:=A^{\prime} \perp\left\langle p^{\prime}, u^{\prime}\right\rangle=\left\langle E^{\prime}, u^{\prime}\right\rangle$ are nonperpendicular $\mathrm{O}^{-}(4, q)$-subspaces such that $U=\langle A, p, u\rangle\left\langle\left\langle A^{\prime}, p^{\prime}\right\rangle^{\perp}=E^{\prime \perp}\right.$ and $U^{\prime}<E^{\perp}$ behave as required.)

If $\Omega$ and $\Omega^{\prime}$ are the sets of singular points of $U$ and $U^{\prime}$, respectively, we claim that

$$
\Omega^{\bullet}:=(\Omega-\{p\}) \cup\left(\Omega^{\prime}-\left\{p^{\prime}\right\}\right) \cup\{x\}
$$

behaves as stated in the lemma. Clearly, $\left|\Omega^{\bullet}\right|=q^{2}+q^{2}+1$.

Orthogonal partial ovoid: $x^{\perp} \cap U=p^{\perp} \cap U=E$ has only one singular point $p$, and $p \notin \Omega^{\bullet}$. Suppose that there are perpendicular singular points $y \in \Omega-E$ and 
$y^{\prime} \in \Omega^{\prime}-E^{\prime}$. Since $y \in U<E^{\prime \perp}$ and $y^{\prime}<E^{\perp}$, while $E$ and $E^{\prime}$ are perpendicular, we obtain to the contradiction that $\langle y, E\rangle=U$ and $\left\langle y^{\prime}, E^{\prime}\right\rangle=U^{\prime}$ are perpendicular.

Maximality: Suppose that $h$ is a singular point such that $h^{\perp} \cap \Omega^{\bullet}=\emptyset$. Then $h^{\perp} \cap U$ is a hyperplane of $U$ and hence contains a singular point, which must be $p$. Then $h^{\perp} \cap U=p^{\perp} \cap U=E$. Also $h^{\perp} \cap U^{\prime}=E^{\prime}$. Now $h \in\left\langle E, E^{\prime}\right\rangle^{\perp}=\left\langle p, p^{\prime}\right\rangle$, which contradicts the assumption that $h$ is not perpendicular to $x \in \Omega^{\bullet}$.

Theorem 7.10. For any $q$ an $\mathrm{O}^{+}(8, q)$-space has a maximal orthogonal partial spread $\Sigma$ of size $2 q^{2}+1$. If $q$ is even then $\Sigma$ is symplectic.

Proof. Applying triality to the lemma proves the first part, while Lemma 4.8 implies the second part.

When $q$ is even, Theorem 5.2(ii) contains another maximal symplectic partial spread of size $2 q^{2}+1$ that need not be orthogonal.

Note that these examples, and others earlier in this section, would have been awkward to describe using t.s. 4-spaces instead of points.

7.3.2. Suzuki-Tits ovoids. Another example of an orthogonal partial ovoid is ( $\Omega-$ $\{p\}) \cup\{x\}$, where $p$ is a point of a Suzuki-Tits ovoid $\Omega$ in an $\mathrm{O}(5, q)$-space $U$ and $x \notin U$ is a singular point in $\left(p^{\perp} \cap U\right)^{\perp}-U^{\perp}$ (see Appendix B). This time it is easier to extend this to a maximal orthogonal partial ovoid of an $O^{+}(8, q)$-space. In the next section we will see further advantages of $\Omega$ over an elliptic quadric.

Theorem 7.11. If $q=2^{2 e+1}>2$ then an $\mathrm{O}^{+}(8, q)$-space has a maximal orthogonal partial spread $\Sigma$ of size $q^{2}+q+1$ that is symplectic.

Proof. By triality and Lemma 4.8, we need to construct a maximal orthogonal partial ovoid of the stated size in an $\mathrm{O}^{+}(8, q)$-space $V$ containing $U$. The radical $r$ of $U$ is also the radical of the 3-space $U^{\perp}$, and $\left(p^{\perp} \cap U\right)^{\perp}=\left\langle p, U^{\perp}\right\rangle=p \perp U^{\perp}$ for $p \in \Omega$. Each singular point in the 4-space $\left(p^{\perp} \cap U\right)^{\perp}$ lies on a t.s. line containing $p$ and meeting $U^{\perp}$ in one of its $q+1$ singular points.

For each singular point $x_{0}$ in $U^{\perp}$ let $x$ be any point in $\left\langle p, x_{0}\right\rangle-\left\{p, x_{0}\right\}$. Let $X$ be the resulting set of $q+1$ points $x$. We claim that

$$
\Omega^{\bullet}:=(\Omega-\{p\}) \cup X
$$

behaves as required. Clearly, $\left|\Omega^{\bullet}\right|=q^{2}+q+1$.

Orthogonal partial ovoid: $x^{\perp} \cap U=p^{\perp} \cap U$ since $x_{0}^{\perp} \geq U$. Then $x^{\perp} \cap \Omega=\{p\}$. No two members of $X$ are perpendicular since no two singular points in $U^{\perp}$ are.

Maximality: Suppose that $h$ is a singular point such that $h^{\perp} \cap \Omega^{\bullet}=\emptyset$. Then $h^{\perp} \cap U$ is a hyperplane of $U$ that cannot contain a point of $\Omega-\{p\}$. By Lemma B.1, $h^{\perp} \geq h^{\perp} \cap U=p^{\perp} \cap U$. Then $h \in\left(p^{\perp} \cap U\right)^{\perp}=\left\langle p, U^{\perp}\right\rangle$, so that $h$ lies on one of the above lines $\left\langle p, x_{0}\right\rangle$, whereas $h^{\perp} \cap X=\emptyset$.

Questions 7.12. Instead of using a single pair $(p, x)$ for replacement what happens if several such pairs are used? Can Section 7.3.1 be handled better than at present in order to use several replacement pairs?

7.4. Small maximal partial spreads. We will describe counterexamples to Grassl's conjecture, which was stated in the Introduction. Grassl $[\mathrm{Gr}]$ has also found counterexamples to his conjecture in an $\mathrm{Sp}(8,8)$-space by a computer search. 
Theorem 7.13. If $q=2^{2 e+1}>2$ then there is a maximal partial $\mathrm{O}^{+}(8, q)$-spread of size $q^{2}-q+1$; this is also a maximal partial $\operatorname{Sp}(8, q)$-spread.

Proof. In view of triality and Lemma 4.8, it suffices to construct a maximal partial $\mathrm{O}^{+}(8, q)$-ovoid of size $q^{2}-q+1$. We use the notation in Section 7.3 .2 and Appendix B]

Let $\Omega$ be a Suzuki-Tits ovoid in an $\mathrm{O}(5, q)$-space $U$. Embed $U$ into an $\mathrm{O}^{+}(8, q)$ space $V$.

Let $\Omega^{\bullet}:=\left(\Omega-\left(x^{\perp} \cap \Omega\right)\right) \cup\{x\}$ for a singular point $x$ of $U$ not in $\Omega$ (this uses $\operatorname{dim} U>4)$. Then $\left|\Omega^{\bullet}\right|=q^{2}-q+1$ and $\Omega^{\bullet}$ is an orthogonal partial ovoid of $U$ and hence of $V$.

Maximality: Suppose that $h$ is a singular point of $V$ such that $h^{\perp} \cap \Omega^{\bullet}=\emptyset$. We will consider the possibilities for the hyperplane $h^{\perp} \cap U$ of $U$ in Lemma B.1. We have $h^{\perp} \cap \Omega \subseteq x^{\perp} \cap \Omega$ since $h^{\perp} \cap \Omega^{\bullet}=\emptyset$. Also, $\Omega^{\perp}=U^{\perp}<x^{\perp}$ since $x \in U=\langle\Omega\rangle$.

Case 1. $h^{\perp} \cap \Omega=\{p\}$ for some $p \in x^{\perp} \cap \Omega$. Then $h^{\perp} \cap U=p^{\perp} \cap U$ since Lemma B.1 implies that $p^{\perp} \cap U$ is the only hyperplane of $U$ meeting $\Omega$ just in $p$. Then $h^{\perp} \geq h^{\perp} \cap U=p^{\perp} \cap U$, so that $h \in\left\langle p, U^{\perp}\right\rangle \leq x^{\perp}$, whereas $h$ is assumed not to be perpendicular to $x \in \Omega^{\bullet}$.

Case 2. $\left|h^{\perp} \cap \Omega\right|=q+1$. Since $h^{\perp} \cap \Omega \subseteq x^{\perp} \cap \Omega$ for sets of size $q+1$, we have $h^{\perp} \geq\left\langle h^{\perp} \cap \Omega\right\rangle=\left\langle x^{\perp} \cap \Omega\right\rangle$, where $\left\langle x^{\perp} \cap \Omega\right\rangle=x^{\perp} \cap U$ by the end of Lemma B.1(ii). Then $h \in\left\langle x, U^{\perp}\right\rangle \leq x^{\perp}$, which produces the same contradiction as before. (This is where an elliptic quadric $\Omega$ would not suffice: we would only have $\left\langle x^{\perp} \cap \Omega\right\rangle<x^{\perp} \cap U$ since $\operatorname{dim} U=5$.)

Case 3. $1<\left|h^{\perp} \cap \Omega\right|<q+1$. Since $h^{\perp} \cap \Omega$ lies in a set $x^{\perp} \cap \Omega$ that projects into a plane of $U / r$, this contradicts the irreducibility in Lemma B.1(iii).

We can go further (mimicking the proofs of Theorems 7.3 and 9.1):

Theorem 7.14. An $\mathrm{O}^{+}(8, q)$-space has a maximal orthogonal partial spread of size $q^{2}-s q+2 s-1$ whenever $q=2^{2 e+1}>2$ and $1<s \leq \sqrt{q / 2}-1$. Each of these is a maximal partial symplectic spread.

In particular, there is a maximal partial $\mathrm{Sp}(8, q)$-spread of size $q^{2}-\sqrt{q^{3} / 2}+q+$ $\sqrt{2 q}-3$.

Proof. Once again we will construct maximal partial $\mathrm{O}^{+}(8, q)$-ovoids. Let $\Omega, U, r$ and $V$ be as before. Choose distinct $a, b \in \Omega$. Then $\{a, b\}^{\perp} \cap U$ is a nondegenerate plane containing $r$. There are $q+1$ singular points $x \in\{a, b\}^{\perp}$. These produce $q+1$ subspaces $\langle x, a, b, r\rangle=x^{\perp} \cap U$ that induce a partition of $\Omega-\{a, b\}$ using the $q+1$ circles $\Omega_{x}:=x^{\perp} \cap \Omega$ of the inversive plane $\mathfrak{I}(\Omega)$ determined by $\Omega$ [De, Sec. 6.4].

Let $\mathcal{X}$ be any set of $s$ singular points $x \in\{a, b\}^{\perp}$. We will show that

$$
\Omega^{\bullet}:=\left(\Omega-\bigcup_{x \in \mathcal{X}} \Omega_{x}\right) \cup \mathcal{X}
$$

is a maximal partial ovoid of the stated size.

1. $\left|\Omega^{\bullet}\right|=\left(q^{2}+1\right)-2-|\mathcal{X}|(q-1)+|\mathcal{X}|$.

2. Partial ovoid: If $x \in \mathcal{X}$ then $\Omega_{x}=x^{\perp} \cap \Omega$ was replaced by $x$, and $\mathcal{X}$ lies in a conic of $\{a, b\}^{\perp} \cap U$.

3. Maximality: Suppose that $h$ is a singular point of $V$ such that $h^{\perp} \cap \Omega^{\bullet}=\emptyset$. Then $h^{\perp} \cap \Omega \subseteq \cup_{x \in \mathcal{X}} \Omega_{x}$. We will consider the various possibilities in Lemma B.1 for the hyperplane $h^{\perp} \cap U$ of $U$. 
Case 1. $h^{\perp} \cap \Omega=\{p\}$ for some $p \in \Omega$. Then $p \in \Omega_{x}$ for some $x \in \mathcal{X} \subset \Omega^{\bullet}$. By Lemma B.1, $h^{\perp} \cap U=p^{\perp} \cap U$. This is inside $h^{\perp}$, so that $h \in\left\langle p, U^{\perp}\right\rangle \leq x^{\perp}$, whereas $h$ is assumed not to be perpendicular to $x \in \Omega^{\bullet}$.

Case 2. $h^{\perp} \cap \Omega$ is a circle. If $h^{\perp} \cap \Omega$ contains $\{a, b\}$ then $h^{\perp} \cap \Omega=\Omega_{x} \subset x^{\perp}$ for some $x \in \mathcal{X}$ since the circles $\Omega_{y}, y \in\{a, b\}^{\perp}$, induce a partition of $\Omega-\{a, b\}$. Then $h^{\perp}$ contains $\left\langle\Omega_{x} \cap U\right\rangle=x^{\perp} \cap U$ by Lemma B.1(ii), which again produces the contradiction $h \in\left\langle x, U^{\perp}\right\rangle \leq x^{\perp}$.

If $h^{\perp} \cap \Omega$ does not contain $\{a, b\}$ then it meets each circle $\Omega_{x}, x \in \mathcal{X}$, in at most two points. This produces the contradiction $q+1=\left|h^{\perp} \cap \Omega\right| \leq 2|\mathcal{X}|=2 s$.

Case 3. $h^{\perp} \cap \Omega$ is an orbit of a cyclic group $T<G$ of order $\left|h^{\perp} \cap \Omega\right|=q \pm \sqrt{2 q}+1$ (Lemma B.1(iii)). We replace the argument used in Theorem 7.13 by counting helped by $T$. Note that $|T|$ divides $q^{2}+1$ and hence is relatively prime to $q(q-1)$, the order of the stabilizer in $G$ of a circle [Su, Theorem 9]. Thus, given circles $C_{1}$ and $C_{2}$, at most one element of $T$ can send $C_{1}$ to $C_{2}$.

For each $t \in T$ we have $h^{\perp} \cap \Omega=\left(h^{\perp} \cap \Omega\right)^{t} \subseteq \cup_{x \in \mathcal{X}} \Omega_{x}^{t}$, involving two sets of $s$ circles: $\left\{\Omega_{x} \mid x \in \mathcal{X}\right\}$ and $\left\{\Omega_{x}^{t} \mid x \in \mathcal{X}\right\}$. For an ordered pair $x, y$ of distinct elements of $\mathcal{X}$ there is at most one such $t \neq 1$ with $\Omega_{x}^{t}=\Omega_{y}$. Thus, if we choose $t$ to be one of at least $|T|-1-s(s-1) \geq q-\sqrt{2 q}-s(s-1)>0$ elements of $T$ that do not behave this way for all $x, y$, then we will have two disjoint sets of $s$ circles, with the union of each set containing $h^{\perp} \cap \Omega$. Since distinct circles meet in at most two points, $q \pm \sqrt{2 q}+1=\left|h^{\perp} \cap \Omega\right| \leq s \cdot s \cdot 2$, which is not the case.

Case $4 . \Lambda:=h^{\perp} \cap \Omega$ has size $q+1$, and its stabilizer in $G$ has a cyclic subgroup $T$ of order $q-1$ having orbit-lengths $1,1, q-1$ on $\Lambda$ (Lemma B.1(iv)). There are $q+1$ orbits of $T$ of size $q-1$; every nontrivial element of $T$ fixes just two points.

If $a$ and $b$ are not the two points fixed by $T$ then it follows that every nontrivial element of $T$ moves every $\Omega_{x}$. Thus, the argument in Case 3 can be repeated (this time with $|T|-1-s(s-1)=q-s(s-1)>0$ and the contradiction $q+1 \leq s \cdot s \cdot 2)$.

If $T$ fixes $a$ and $b$ then $\Lambda$ is not one of the circles $\Omega_{y}$ by Lemma B.1(iv). Note that $T$ fixes two circles containing $\{a, b\}$ and is transitive on the remaining $q-1$ circles containing $\{a, b\}$. Thus, if a nontrivial element of $T$ fixes $\Omega_{y}$ then $\Omega_{y}-\{a, b\}$ is an orbit of $T$, while $\Lambda-\{a, b\}$ is a different orbit, so that $\Omega_{y}$ can be deleted in our union (of $\Omega_{x}, x \in \mathcal{X}$ ) that contains $\Lambda$. Since $T$ is transitive on the set of $q-1$ circles $\Omega_{x}$ that it does not fix, we obtain the contradiction $s=|\mathcal{X}| \geq|T|=q-1$. (Alternatively, the argument in Case 3 can be repeated again.)

We have proved, more generally, that $\Omega^{\bullet}$ is a maximal partial ovoid of any nonsingular orthogonal $\mathbb{F}_{q}$-space containing $U$ since every hyperplane of $U$ has nonempty intersection with $\Omega^{\bullet}$ (cf. Question 7.6).

\section{5. $\operatorname{Sp}(6, q)$-space consequences.}

Theorem 7.15. For even $q>2$, an $\mathrm{Sp}(6, q)$-space has maximal symplectic partial spreads of size

(i) $n_{1}=q^{3}-q^{2}+1$,

(ii) $q^{2}+1$

(iii) $2 q^{2}+1$,

(iv) $q^{2}+q+1$ if $q=2^{2 e+1}$,

(v) $q^{2}-q+1$ if $q=2^{2 e+1}$,

(vi) $q^{2}-s q+2 s-1$ if $q=2^{2 e+1}$ and $1 \leq s \leq \sqrt{q / 2}-1$, 
(vii) $n_{r}$ if $1 \leq r \leq q / 5$ (where $n_{r}$ is defined in Theorem 7.3), and

(vii) $n_{4}-1$ if $q \geq 16$.

Proof. Use Lemma 6.1(i) together with Theorems 7.2, 77.7, 7.10, 7.11, 7.13, 7.14 and 7.3 ,

Example 7.16. By Lemma 6.1(ii), the set of sizes of maximal partial $\mathrm{Sp}(6,4)$ spreads is contained in the set of sizes of maximal partial $\operatorname{Sp}(8,4)$-spreads. This can be compared with the list in $\mathrm{Gr}$.

\section{6-DIMENSIONAL PARTIAL SPREADS}

We again consider arbitrary characteristic. In characteristic 2 the examples in the next theorem already appear in Theorem $\mathbf{7 . 1 5}$ (i) but using an entirely different method to prove maximality.

Theorem 8.1. If $q$ is a prime power then an $\mathrm{Sp}(6, q)$-space has a maximal symplectic partial spread of size $q^{3}-q^{2}+1$.

Proof. In an $\operatorname{Sp}(6, q)$-space let $\Sigma$ be a desarguesian spread preserved by $G=$ $\mathrm{SL}\left(2, q^{3}\right)=\mathrm{Sp}\left(2, q^{3}\right)<\mathrm{Sp}(6, q)$. Let $X \in \Sigma$. Let $U$ be a t.i. 3 -space such that $U \cap X=L$ is a line. If $\Sigma_{U}$ is the set of members of $\Sigma$ met nontrivially by $U$, then we will show that $\Sigma^{\bullet}:=\left(\Sigma-\Sigma_{U}\right) \cup\{U\}$ is a maximal symplectic partial spread of size $q^{3}-q^{2}+1$.

If $U$ meets $Y \in \Sigma-\{X\}$ nontrivially then $U \cap Y$ must meet $U \cap X=L$ trivially and hence is a point; the number of such points is the number $q^{2}$ of points in $U$ not in $L$. Thus, $\left|\Sigma_{U}\right|=q^{2}+1$ and $\Sigma^{\bullet}$ is a symplectic partial spread of size $q^{3}-q^{2}+1$.

The set-stabilizer $G_{X}$ of $X$ has order $q^{3}\left(q^{3}-1\right)$, with an abelian normal subgroup $Q$ of order $q^{3}$ inducing 1 on $X$ and a cyclic subgroup $S$ of order $q^{3}-1$ that is transitive on both $X-\{0\}$ and the $q^{2}+q+1$ lines $L$ of $X$. Then $\left|G_{L}\right|=q^{3}(q-1)$. Since $Q$ is transitive on the $q$ t.i. 3 -spaces $\neq X$ containing $L,\left|G_{U}\right|=q^{2}(q-1)$ and $Q_{U}$ fixes each of those 3 -spaces.

Since $S$ is transitive on both the $q^{2}+q+1$ lines $L$ of $X$ the $G_{X}$-conjugates of $Q_{U}$, we obtain a bijection $L \mapsto Q_{U}$ between these sets.

Also $Q$ is transitive on the $q^{3}$ points in $\left\{L^{\perp} \cap Y \mid Y \in \Sigma-\{X\}\right\}$. Since $U$ contains $q^{2}$ of these points, and each such point and $L$ generate $U$, it follows that $Q_{U}$ is transitive on these $q^{2}$ points. Since $Q_{U}$ fixes each t.i. 3-space containing $L$, the $q$ orbits of $Q_{U}$ partitioning $\Sigma-\{X\}$ correspond to the $q$ t.i. 3-spaces $\neq X$ containing $L$.

Maximality: Assume that $W \notin \Sigma^{\bullet}$ is a t.i. 3-space such that $\Sigma^{\bullet} \cup\{W\}$ is a symplectic partial spread. Then $\Sigma_{W} \subseteq \Sigma_{U}$ since $\Sigma$ is a spread. Clearly, $W$ meets each member of $\Sigma$ in 0 , a point or a line. Since $\left|\Sigma_{W}\right| \leq\left|\Sigma_{U}\right|=q^{2}+1$, some intersection is a line, and it is unique (since two lines of $W$ would meet nontrivially). Thus, $W$ arises in the same manner as $U$, and $G_{W}$ acts on $\Sigma_{W}=\Sigma_{U}$.

We cannot have $L=X \cap U=X \cap W$ in view of the above orbit partition, and we cannot have $G_{U}=G_{W}$ in view of the above bijection. Then $\left\langle G_{U}, G_{W}\right\rangle$ is generated by distinct subgroups of $G$ of order $q^{2}(q-1)$, and hence has a subgroup of order $q^{3}$ with an orbit on $\Sigma$ of size $q^{3}$. (This is a very special case of 115 -year-old group theory summarized in [Dic, Ch. XII].) This contradicts the fact that $\left|\Sigma_{U}\right|<q^{3}$. 


\section{4-DIMENSIONAL PARTIAL SPREADS}

Finally, we survey families of maximal partial spreads of $\operatorname{Sp}(4, q)$-spaces. See CDFS, Gr for lists and tables of known families. As suggested in Section 1, we can use more easily visualized points in $\mathrm{O}(5, q)$-space instead of lines in $\operatorname{Sp}(4, q)$ space due to the Klein correspondence [Ta, p. 196].

Theorem 9.1 ([CDFS, p. 1940], [ThK, Theorem 6.6]).

(i) For odd $q$ an $\mathrm{Sp}(4, q)$-space has a maximal partial spread of size $q^{2}-s q+$ $3 s-1$ whenever $1 \leq s<(q+1) / 2$.

(ii) For even $q$ an $\operatorname{Sp}(4, q)$-space has a maximal partial spread of size $q^{2}-s q+$ $2 s-1$ whenever $1 \leq s<(q+1) / 2$.

Proof. We will construct maximal partial $\mathrm{O}(5, q)$-ovoids. Start with an $\mathrm{O}^{-}(4, q)$ ovoid $\Omega$ in a 4-dimensional subspace $U$. Choose distinct $a, b \in \Omega$. If $y$ is a singular point not in $\Omega$, then $y^{\perp} \cap \Omega$ is an oval (more precisely, a conic in $\left\langle y^{\perp} \cap \Omega\right\rangle$ ).

(i) The planes $E$ of $U$ containing $\{a, b\}$ fall into two sets $\Pi_{k}, k=0$ or 2, each of size $(q+1) / 2$, such that the nonsingular line $E^{\perp}$ has exactly $k$ singular points. Let $\Omega_{E}:=E \cap \Omega$. With each $E \in \Pi_{2}$ is an associated $E^{\prime} \in \Pi_{2}$ (for an involution $E \mapsto E^{\prime}$ without fixed points) such that there are exactly two singular points $x_{E}, x_{E^{\prime}}$ in $E \cap\{a, b\}^{\perp}$ and $(*) \Omega_{E}=x_{E}^{\perp} \cap \Omega=x_{E^{\prime}}^{\perp} \cap \Omega=\Omega_{E^{\prime}}$. Then $\left\{x_{E}, x_{E^{\prime}} \mid E \in \Pi_{2}\right\}$ lies in a conic in the plane $\{a, b\}^{\perp}$. The members of $\Pi_{0} \cup \Pi_{2}$ induce a partition of $\Omega-\{a, b\}$.

Let $\mathcal{S}$ be any set of $s<(q+1) / 2$ planes $E \in \Pi_{2}$ such that the conics $\Omega_{E}, E \in \mathcal{S}$, are distinct $($ cf. $(*))$. We claim that $\Omega^{\bullet}:=\left(\Omega-\bigcup_{E \in \mathcal{S}} \Omega_{E}\right) \cup \bigcup_{E \in \mathcal{S}}\left\{x_{E}, x_{E^{\prime}}\right\}$ is a maximal partial $\mathrm{O}(5, q)$-ovoid of the stated size.

1. $\left|\Omega^{\bullet}\right|=\left(q^{2}+1\right)-2-|\mathcal{S}|(q-1)+2|\mathcal{S}|$.

2. Orthogonal partial ovoid: If $E \in \mathcal{S}$ then $\Omega_{E}=x_{E}^{\perp} \cap \Omega=x_{E^{\prime}}^{\perp} \cap \Omega$ was replaced by $\left\{x_{E}, x_{E^{\prime}}\right\}$, lying in a conic of $\{a, b\}^{\perp}$.

3. Maximality: Every point of $\Omega$ is either in $\Omega^{\bullet}$ or is perpendicular to $\left\{x_{E}, x_{E^{\prime}}\right\}$ for some $E \in \mathcal{S}$. Suppose that $h \notin \Omega$ is a singular point such that $h^{\perp} \cap \Omega^{\bullet}=\emptyset$. Then $h^{\perp} \cap \Omega \subseteq \bigcup_{E \in \mathcal{S}} \Omega_{E}$ and $h^{\perp} \cap \Omega$ is either a point or a circle of the inversive plane $\Im(\Omega)$ determined by $\Omega$ [De, Sec. 6.4] (compare Example 7.4).

If $h^{\perp} \cap \Omega$ is a point $p$ then $h^{\perp} \cap U$ is the tangent plane to $\Omega$ at $p$ in $U$. Then $h \in\left(h^{\perp} \cap U\right)^{\perp}=\left(p^{\perp} \cap U\right)^{\perp}=\left\langle p, U^{\perp}\right\rangle$, which has just one singular point $p$, whereas $h \notin \Omega$. Thus, $h^{\perp} \cap \Omega \subseteq \bigcup_{E \in \mathcal{S}} \Omega_{E}$ is a circle.

If $h^{\perp} \cap \Omega=\Omega_{E}$ with $E \in \mathcal{S}$, then $h \in\left(\Omega_{E}\right)^{\perp}=E^{\perp}=\left\langle x_{E}, x_{E^{\prime}}\right\rangle$, whereas $h \notin\left\{x_{E}, x_{E^{\prime}}\right\}$. Thus, $h^{\perp} \cap \Omega$ is a circle lying in the union of $s$ other circles, each of which it meets at most twice. This produces the contradiction $q+1=\left|h^{\perp} \cap \Omega\right| \leq 2 s$.

(ii) This is proved as above but is simpler: $\left(E^{\perp} \cap \Omega\right)^{\perp}$ contains just one singular point for each plane $E$ of $U$ containing $\{a, b\}$; no permutation $E \mapsto E^{\prime}$ is involved.

It is tempting to hope that the above argument only used an $\mathrm{O}(5, q)$-ovoid $\Omega$. However, when $q$ is odd the intersections $x^{\perp} \cap \Omega$ are not sufficiently well-behaved. When $q$ is even Section 7.4 contains versions of the preceding argument in $\mathrm{O}^{+}(8, q)$ space (also compare Theorem 7.3 ).

Example 9.2. A maximal partial ovoid of size $3 q-1$ in $\operatorname{Sp}(4, q)$-space, $q \geq 4$, is constructed in [CDFS, p. 1939]. The proof in that paper shows that this is a maximal partial ovoid in $\operatorname{Sp}(2 m, q)$-space for all $m \geq 2$. 
This partial ovoid is the set of points in $\bigcup_{1}^{3}\left(\left\langle x_{i}, y_{i+1}\right\rangle-\left\{x_{i}, y_{i+1}\right\}\right) \cup\{x, y\}$ (subscripts mod 3), where $x_{1}, x_{2}, x_{3}, x$ are four points of $X$ and $y_{1}, y_{2}, y_{3}, y$ are four points of $Y$ for t.i. 2-spaces $X, Y$ intersecting in 0 , with each pair $x_{i}, y_{i}$ perpendicular and $x, y$ not perpendicular.

Dualizing [Ta, p. 196] produces a maximal symplectic partial spread of size $3 q-1$ in $\operatorname{Sp}(4, q)$-space for even $q \geq 4$.

Example 9.3. For arbitrary $q$, [RS, [PRS] has integer intervals that consist of sizes of maximal partial $\mathrm{Sp}(4, q)$-spreads.

Example 9.4. There is a maximal partial spread of size $q^{2}-1$ in $\operatorname{Sp}(4, q)$-space for $q \in\{3,5,7,11\}$. This is constructed using a subgroup of $\operatorname{Sp}(2, q)=\operatorname{SL}(2, q)$ sharply transitive on $\mathbb{F}_{q}^{2}-\{0\}[\mathrm{Pe}, \overline{\mathrm{DH}}, \mathrm{CDS}$. It is contained in the non-symplectic spread of $\mathbb{F}_{q}^{4}$ corresponding to the associated affine irregular nearfield plane.

\section{CONCLUding REMARKS}

The preceding examples makes it clear that there are rather few known types of maximal symplectic partial spreads. There are amazingly few known types in odd characteristic, especially in view of the tables in CDFS, $\mathrm{Gr}$. We mentioned a number of symplectic partial spreads whose maximality has yet to be decided.

We have not yet considered most inequivalence questions for given dimension and field size. Suppose that $q$ is even. The number of inequivalent orthogonal spreads in $\mathrm{O}^{+}(4 m, q)$-spaces is not bounded above by any polynomial in $q^{m}[\mathrm{KaW}]$; these produce inequivalent maximal symplectic partial spreads in Proposition 4.1. 4.5 and 6.3. In addition, there are at least $q^{q^{k}} / q^{4 k^{2}}$ inequivalent examples in Theorem 5.2 (ii), $\left(\begin{array}{c}q-1 \\ s\end{array}\right) / q^{30}$ for each pair $q, s$ in Theorem $7.3,(q-1)^{q+1} / q^{30}$ in Theorem 7.11, $\left(\begin{array}{c}q+1 \\ s\end{array}\right) / q^{30}$ for each pair $q, s$ in Theorem $7.14,\left(\begin{array}{c}q+1 \\ s\end{array}\right) / q^{11}$ for each pair $q, s$ in Theorem 9.1 and $(q-2)(q-3) / 6 \log q$ in Example 9.2 .

Acknowledgements. I am grateful to Markus Grassl for stimulating my interest in maximal symplectic partial spreads by pointing out the scarcity of examples in dimension $>4$. This research was supported in part by a grant from the Simons Foundation.

\section{REFERENCES}

[BS1] B. Bagchi and N. S. N. Sastry, Even order inversive planes, generalized quadrangles and codes. Geom. Ded. 22 (1987) 137-147.

[BS2] B. Bagchi and N. S. N. Sastry, Intersection pattern of the classical ovoids in symplectic 3-space of even order. J. Algebra 126 (1989) 147-160.

[Ba] S. Ball, On ovoids of O $(5, q)$. Adv. Geom. 4 (2004) 1-7.

[CDFS] M. Cimráková, S. De Winter, V. Fack and L. Storme, On the smallest maximal partial ovoids and spreads of the generalized quadrangles $W(q)$ and $Q(4, q)$. Eur. J. Comb. 28 (2007) 1934-1942.

[CDS] K. Coolsaet, J. De Beule and A. Siciliano, The known maximal partial ovoids of size $q^{2}-1$ of $Q(4, q)$. J. Comb. Des. 21 (2013) 89-100.

[Co] B. N. Cooperstein, Hyperplane sections of Kantor's unitary ovoids. Des. Codes Cryptogr. 23 (2001) 185-195.

[DH] W. van Dam and M. Howard, Bipartite entangled stabilizer mutually unbiased bases as maximum cliques of Cayley graphs. Phys. Rev. A 84 (2011) 012117.

[De] P. Dembowski, Finite Geometries. Springer, Berlin 1968; reprinted, 1997.

[Dic] L. E. Dickson, Linear groups with an exposition of the Galois field theory. Teubner, Leipzig 1901; reprinted, Dover, New York 1958. 
[Dil] J. F. Dillon, Elementary Hadamard difference sets. Ph.D. thesis, U. of Maryland 1974.

[Dye] R. H. Dye, Partitions and their stabilizers for line complexes and quadrics. Ann. Mat. Pura Appl. 114 (1977) 173-194.

[Gr] M. Grassl, Unextendible sets of mutually unbiased bases (MUBs), talk at "Systems of Lines, Applications of Algebraic Combinatorics", Conference at Worcester Polytechnic Institute, August 10-14, 2015.

[HB] B. Huppert and N. Blackburn, Finite groups III. Springer, Berlin-New York 1982.

[Ka1] W. M. Kantor, Ovoids and translation planes. Canad. J. Math. 34 (1982) 1195-1207.

[Ka2] W. M. Kantor, Codes, quadratic forms and finite geometries, pp. 153-177 in: Different aspects of coding theory (Ed. A. R. Calderbank), Proc. AMS Symp. Applied Math. 50, AMS, Providence 1995.

[Ka3] W. M. Kantor, MUBs and affine planes. J. Mathematical Physics 53 (2012) 032204

$[\mathrm{KaW}]$ W. M. Kantor and M. E. Williams, Symplectic semifield planes and $\mathbb{Z}_{4}$-linear codes. TAMS 356 (2004) 895-938.

[MBGW] P. Mandayam, S. Bandyopadhyay, M. Grassl and W. K. Wootters, Unextendible mutually unbiased bases from Pauli classes. Quantum Inf. Comput. 14 (2014) 823-844.

[Pe] T. Penttila (unpublished).

[PW] T. Penttila and B. Williams, Ovoids of parabolic spaces. Geom. Ded. 82 (2000) 1-19.

[PRS] V. Pepe, C. Rößing and L. Storme, A spectrum result on maximal partial ovoids of the generalized quadrangle $Q(4, q), q$ odd, pp 349-362 in: Finite fields: theory and applications. Contemp. Math. 518, AMS, Providence 2010.

[RS] C. Rößing and L. Storme, A spectrum result on maximal partial ovoids of the generalized quadrangle $Q(4, q), q$ even. Eur. J. Comb. 31 (2010) 349-361.

[Su] M. Suzuki, On a class of doubly transitive groups. Ann. of Math. 75 (1962) 105-145.

[Ta] D. E. Taylor, The geometry of the classical groups. Heldermann, Berlin 1992.

[Th] J. A. Thas, Old and new results on spreads and ovoids of finite classical polar spaces. Combinatorics '90 (Gaeta, 1990). Ann. Disc. Math. 52 (1992) 529-544.

[ThK] K. Thas, Unextendible mutually unbiased bases (after Mandayam, Bandyopadhyay, Grassl and Wootters). arXiv:1407.2778v1.

[Ti1] J. Tits, Sur la trialité et certains groupes qui s'en déduisent. Publ. Math. I.H.E.S. 2 (1959) 13-60.

[Ti2] J. Tits, Ovoïdes et groupes de Suzuki. Arch. Math. 13 (1962) 187-198.

[Ti3] J. Tits, Ovoïdes à translations. Rend. Mat. e Appl. 21 (1962) 37-59.

\section{Appendix A. The desarguesian ovoid in $\mathrm{O}^{+}(8, q)$-Space}

In order to prove Theorem 7.3 we will consider a specific orthogonal ovoid in an $\mathrm{O}^{+}(8, q)$-space with $q>2$ even. Let $F=\mathbb{F}_{q^{3}} \supset K=\mathbb{F}_{q}$, with trace map $T: F \rightarrow K$ and norm $N: F \rightarrow K$. Then $Q(a, \beta, \gamma, d):=a d+T(\beta \gamma)$ turns $V:=K \oplus F \oplus F \oplus K$ into an $\mathrm{O}^{+}(8, q)$-space.

The $q^{3}+1$ points $\langle(0,0,0,1)\rangle$ and $\left\langle\left(1, t, t^{q+q^{2}}, N(t)\right\rangle, t \in F\right.$, form an ovoid $\Omega$ on which $G:=\operatorname{SL}\left(2, q^{3}\right)$ acting 3 -transitively. In [Ka1, p. 1204] this is called a desarguesian ovoid (since it arises from a desarguesian spread of an $\operatorname{Sp}(6, q)$-space using Lemma 6.1(ii) and triality), and it is observed that $G$ has exactly two orbits of singular points of $V$, one of which is $\Omega$. If $q>2$ and $p$ is any singular point not in $\Omega$, then $\left\langle p^{\perp} \cap \Omega\right\rangle=p^{\perp}$ [Ka1, p. 1204], as required in Lemma 7.1

Notation A.1. Let $\pi \in F$ with $T(\pi)=0 \neq T\left(\pi^{1+q}\right)$. Use the nondegenerate symmetric $K$-bilinear form $T(x y)$ on $F$ to see that $\pi^{q} \notin\{t \in F \mid T(\pi t)=0\}=$ $K+K \pi$.

Lemma A.2. If $p_{1}$ and $p_{2}$ are distinct singular points not in $\Omega$, then $\left|p_{1}^{\perp} \cap p_{2}^{\perp} \cap \Omega\right| \leq$ $5 q-5$.

Proof. By the transitivity of $G$ we may assume that $p_{1}=\langle(0,0, \pi, 0)\rangle$ and $p_{2}=$ $\langle(a, \beta, \gamma, d)\rangle$ for some $a, \beta, \gamma, d$. We need to estimate the number of solutions $t$ to 
the equations

$$
T(t \pi)=0=a N(t)+d+T\left(\beta t^{q+q^{2}}+\gamma t\right)
$$

corresponding to points $\left\langle\left(1, t, t^{q+q^{2}}, t^{1+q+q^{2}}\right)\right\rangle$. By A.1 we can write $t=u+v \pi$ with $u, v \in K$. Then the second equation is

$$
a N(u+v \pi)+d+T\left(\beta[u+v \pi]^{q+q^{2}}+\gamma[u+v \pi]\right)=0,
$$

which expands to

$$
\begin{aligned}
a\left\{u^{3}\right. & \left.+u v^{2} T\left(\pi^{q+q^{2}}\right)+v^{3} N(\pi)\right\}+d \\
& +u^{2} T(\beta)+u v T(\beta \pi)+v^{2} T\left(\beta \pi^{q+q^{2}}\right)+u T(\gamma)+v T(\gamma \pi)=0 .
\end{aligned}
$$

For each $u$ this is a $K$-polynomial in $v$ of degree at most three, and hence has at most three roots $v \in K$ if it is not the zero polynomial. Let $B$ be the number of "bad" $u$ for which this polynomial in $v$ is the zero polynomial. Then $\left|p_{1}^{\perp} \cap p_{2}^{\perp} \cap \Omega\right| \leq$ $(q-B) 3+B q+1$ (the last term occurs since $\langle(0,0,0,1)\rangle$ may be in the intersection). We will show that $B \leq 2$, which produces the bound in the lemma.

The coefficients of our polynomial show that, for a "bad" $u$, we must have $a=0$, then $T\left(\beta \pi^{q+q^{2}}\right)=0, u T(\beta \pi)+T(\gamma \pi)=0$ and $u^{2} T(\beta)+u T(\gamma)+d=0$. If $T(\beta \pi) \neq 0$ then there is one "bad" $u$, and if $T(\beta \pi)=T(\gamma \pi)=0$ then there are at most two "bad" $u$ unless $T(\beta)=T(\gamma)=d=0$.

Thus, we must show that $T\left(\beta \pi^{q+q^{2}}\right)=T(\beta \pi)=T(\gamma \pi)=T(\beta)=T(\gamma)=0$ cannot all occur. Since $T(\beta)=T(\beta \pi)=0$, by A.1 we have $\beta=x \pi$ with $x \in K$. Then $0=T\left(\beta \pi^{q+q^{2}}\right)=x T(N(\pi))$, so that $x=0$. Similarly, $T(\gamma)=T(\gamma \pi)=0$ implies that $\gamma=y \pi$ with $y \in K$. Now $p_{2}=\langle(0,0, y \pi, 0)\rangle=p_{1}$, which is not the case.

Notation A.4. Let $\Omega_{0} \subset \Omega$ consist of $\langle(0,0,0,1)\rangle$ and $\left\langle\left(1, t, t^{q+q^{2}}, t^{1+q+q^{2}}\right)\right\rangle, t \in K$. There are $(q+1)^{2}$ singular points in $\Omega_{0}^{\perp}$, all having the form $\langle(0, \beta, \gamma, 0)\rangle$ with $T(\beta)=T(\gamma)=T(\beta \gamma)=0$. The sets $\Omega_{0}$ and $\Omega_{0}^{\perp}$ are acted on by a naturally embedded subgroup $G_{0}=\operatorname{SL}(2, q)$ of $G$ containing the transformations

$$
\begin{aligned}
u_{s}:(a, \beta, \gamma, d) & \mapsto\left(a, \beta+s a, \gamma+a s^{2}+\beta^{q} s+\beta^{q^{2}} s, d+a s^{3}+T(\beta) s^{2}+T(\gamma) s\right) \\
j:(a, \beta, \gamma, d) & \mapsto(d, \gamma, \beta, a) .
\end{aligned}
$$

with $s \in K$. These act on each of the $q+1$ lines $\langle(0, \beta, 0,0),(0,0, \beta, 0)\rangle$ with $T(\beta)=0 \neq \beta$ that partition the $(q+1)^{2}$ singular points in $\Omega_{0}^{\perp}$, sending

$$
\begin{aligned}
u_{s}:(0, \beta, \gamma, 0) & \mapsto(0, \beta, \gamma+\beta s, 0) \\
j:(0, \beta, \gamma, 0) & \mapsto(0, \gamma, \beta, 0) .
\end{aligned}
$$

Definition A.6. An ordinary point is a singular point in $\Omega_{0}^{\perp}$ of the form $\langle(0, \beta, \gamma, 0)\rangle$ such that either $\beta=0$ and $T\left(\gamma^{1+q}\right) \neq 0$, or $T\left(\beta^{1+q}\right) \neq 0$ (recall that $T(\beta)=T(\gamma)=$ $T(\beta \gamma)=0)$. Since any $\beta \in F^{*}$ has characteristic polynomial $x^{3}+T(\beta) x^{2}+T\left(\beta^{1+q}\right)+$ $N(\beta)$, the ordinary requirement can fail for some $\beta, \gamma$ if and only if $q \equiv 1$ (mod $3)$. Moreover, if $\beta \in F-K$ then $\beta^{q} \in \beta K \Longleftrightarrow \beta^{q-1} \in K \Longleftrightarrow \beta^{\left(q-1, q^{2}+q+1\right)} \in$ $K \Longleftrightarrow \beta^{3} \in K \Longleftrightarrow T\left(\beta^{1+q}\right)=0$.

For $\pi$ in (A.1), since $T\left(\left(a \pi+\pi^{q}\right)\left(a \pi+\pi^{q}\right)^{q}\right)=\left(a^{2}+a+1\right) T\left(\pi^{1+q}\right)$ the points of the line $\left\langle\left(0, a \pi+\pi^{q}, 0,0\right),\left(0,0, a \pi+\pi^{q}, 0\right)\right\rangle, a \in K$, are ordinary if and only if $a^{2}+a+1 \neq 0$, so that all points are ordinary if $q \equiv 2(\bmod 3)$, but there are two lines of this form all of whose points are not ordinary when $q \equiv 1(\bmod 3)$.

The significance of ordinary points is the following

Lemma A.7. If $p$ is an ordinary point then 
(i) $p$ has the form $\langle(0,0, \gamma, 0)\rangle$ with $T(\gamma)=0$ or $\langle(0, \beta, a \beta, 0)\rangle$ with $T(\beta)=0$ and $a \in K$, and

(ii) $p^{g}=\left\langle\left(0,0, \pi^{\prime}, 0\right)\right\rangle$ for some $g \in G_{0}$, where $\pi^{\prime}$ behaves as $\pi$ does in (A.1): $T\left(\pi^{\prime}\right)=0 \neq T\left(\pi^{\prime 1+q}\right)$.

Proof. We may assume that $p=\langle(0, \beta, \gamma, 0)\rangle$ with $\beta \neq 0$.

(i) Since $p$ is ordinary, $\beta^{q} \notin K \beta$, so that $\beta$ and $\beta^{q}$ span $\operatorname{ker} T$. Write $\gamma=k \beta+b \beta^{q}$ with $k, b \in K$. Then $0=T(\beta \gamma)=b T\left(\beta^{1+q}\right)$ implies that $b=0$.

(ii) By (A.5), $p^{u_{k} j}=\langle(0,0, \beta, 0)\rangle$ behaves as stated.

Lemma A.8. If $p_{1}, p_{2}$, and $p_{3}$ are pairwise non-perpendicular ordinary points, then

(i) $\left|p_{1}^{\perp} \cap p_{2}^{\perp} \cap \Omega\right|=2 q$ and

(ii) $\left|p_{1}^{\perp} \cap p_{2}^{\perp} \cap p_{3}^{\perp} \cap \Omega\right|=q+2$.

Proof. By Lemma A.7(ii) we may assume that $p_{1}$ has the form $\langle(0,0, \pi, 0)\rangle$ and $p_{2}=\langle(0, \beta, \gamma, 0)\rangle$, where $T(\beta)=T(\gamma)=T(\beta \gamma)=0$. Also $T(\beta \pi) \neq 0$ since $p_{1}$ and $p_{2}$ are not perpendicular. All $(0,0,0,1)$ and $\left(1, t, t^{q+q^{2}}, N(t)\right), t \in K$, are in each of the stated intersections, so we will focus on vectors $\left(1, t, t^{q+q^{2}}, N(t)\right)$ with $t=u+v \pi \notin K$ in the intersections.

(i) Here (A.3) states that

$$
u v T(\beta \pi)+v^{2} T\left(\beta \pi^{q+q^{2}}\right)+v T(\gamma \pi)=0 .
$$

Since $T(\beta \pi) \neq 0$, each $v \neq 0$ determines a unique $u$. This argument reverses: the intersection size is $(q+1)+(q-1)$.

Before continuing we massage (A.9). By Lemma A.7(i), $\gamma=k \beta$ for some $k \in K$. Since $\operatorname{dim} \operatorname{ker} T=2$ we can write $\beta=x \pi+y \pi^{q}$ with $x, y \in K$. Since $0 \neq T(\beta \pi)=$ $y T\left(\pi^{1+q}\right)$ we have $y \neq 0$ and $\beta \in\left((x / y) \pi+\pi^{q}\right) K$. We may assume that $\beta=a \pi+\pi^{q}$ with $a \in K$. Then

$$
p_{2}=\left\langle\left(0, a \pi+\pi^{q}, k\left(a \pi+\pi^{q}\right), 0\right)\right\rangle,
$$

so that $T(\beta \pi)=T\left(\pi^{1+q}\right)$ and (A.9) becomes

$$
u T\left(\pi^{1+q}\right)+v\left[a N(\pi)+T\left(\pi^{2 q+q^{2}}\right)\right]+k T\left(\pi^{1+q}\right)=0 .
$$

(ii) We may assume that $p_{3}=\left\langle\left(0, \beta^{\prime}, \gamma^{\prime}, 0\right\rangle\right)$ with $\gamma^{\prime}=k^{\prime} \beta^{\prime}$ and $\beta^{\prime}=a^{\prime} \pi+\pi^{q}$ for some $k^{\prime}, a^{\prime} \in K$. Then $\left(a+a^{\prime}\right)\left(k+k^{\prime}\right) T\left(\pi \pi^{q}\right)=T\left(\beta \gamma^{\prime}+\gamma \beta^{\prime}\right) \neq 0$. The two versions of (A.11) imply that

$$
v=\frac{k+k^{\prime}}{a+a^{\prime}} \frac{T\left(\pi^{1+q}\right)}{N(\pi)}, \quad u=k+\frac{k+k^{\prime}}{a+a^{\prime}}\left(a+\frac{T\left(\pi^{2 q+q^{2}}\right)}{N(\pi)}\right),
$$

which proves (ii).

Example A.13. (i) If $\mathcal{P} \subseteq\left\{\langle(0,0, \pi, 0)\rangle,\left\langle\left(0, a \pi+\pi^{q}, a^{2} \pi+a \pi^{q}, 0\right)\right\rangle \mid a \in K, a^{2}+\right.$ $a+1 \neq 0\}$, then

$$
\left|\bigcap_{p \in \mathcal{P}} p^{\perp} \cap \Omega\right|= \begin{cases}q^{2}+1 & \text { if }|\mathcal{P}|=1 \\ 2 q & \text { if }|\mathcal{P}|=2 \\ q+2 & \text { if }|\mathcal{P}| \geq 3 .\end{cases}
$$

(ii) If $\mathcal{P} \subseteq\left\{\langle(0,0, \pi, 0)\rangle,\left\langle\left(0, \pi^{q}, 0,0\right)\right\rangle,\left\langle\left(0, \pi+\pi^{q}, \pi+\pi^{q}, 0\right)\right\rangle,\left\langle\left(0, a \pi+\pi^{q}, a^{3} \pi+\right.\right.\right.$ $\left.\left.\left.a^{2} \pi^{q}, 0\right)\right\rangle\right\}$ for an arbitrary $a \in K-\{0,1\}$ such that $a^{2}+a+1 \neq 0$, then 


$$
\left|\bigcap_{p \in \mathcal{P}} p^{\perp} \cap \Omega\right|= \begin{cases}q^{2}+1 & \text { if }|\mathcal{P}|=1 \\ 2 q & \text { if }|\mathcal{P}|=2 \\ q+2 & \text { if }|\mathcal{P}|=3 \\ q+1 & \text { if }|\mathcal{P}|=4\end{cases}
$$

Proof. All of the stated points are ordinary.

(i) In (A.10), $k=a$ for all listed points other than $\langle(0,0, \pi, 0)\rangle$. By (A.12), $t=\frac{T\left(\pi^{2 q+q^{2}}\right)}{N(\pi)}+\frac{T\left(\pi^{1+q}\right)}{N(\pi)} \pi$ is in every intersection (which is easily checked directly); so is $\Omega_{0}$, so that every intersection has size $\geq q+2$. Since any intersection of three sets $p^{\perp} \cap \Omega$ has size $q+2$ (by Lemma A.8(ii)), so does any intersection of at least four such sets.

(ii) The last three of these four ordinary points correspond to the pairs $(a, k)=$ $(0,0),(1,1),\left(a, a^{2}\right)$ in A.10). Then A.12 and different 3 -sets in $\mathcal{P}$ produce different values of $v$, so that $\left|\bigcap_{p \in \mathcal{P}} p^{\perp} \cap \Omega\right|=q+1$ if $|\mathcal{P}|=4$. The remaining sizes are given in Lemma A.8.

\section{Appendix B. Suzuki-Tits ovoids: BaCkground}

We will need information concerning a Suzuki-Tits ovoid $\Omega$ in an $\mathrm{O}(5, q)$-space $U$ with radical $r$, where $q=2^{2 e+1}$. The standard view of these ovoids is in symplectic space. For our purposes, the view from an $\mathrm{O}(5, q)$-space has advantages, such as lying in an $\mathrm{O}^{+}(8, q)$-space.

Let $\bar{\Omega}$ denote a standard Suzuki-Tits ovoid in the symplectic 4-space $U / r$ Ti2. If $\langle x, r\rangle / r \in \bar{\Omega}$ then the line $\langle x, r\rangle$ has a unique singular point. Thus, there is a set $\Omega$ of $q^{2}+1$ singular points of $U$ that projects onto $\bar{\Omega}$. The group $\operatorname{Sz}(q)$ lifts from a subgroup of $\operatorname{Sp}(4, q)$ to a group $G<\mathrm{O}(5, q)$ preserving $\Omega$. See [De, Sec. 6.4] for information concerning the inversive plane $\mathfrak{I}(\Omega)$ produced by $\Omega$.

We will assume that $q>2$. Then $U=\langle\Omega\rangle$ since $G$ does not act on an $\mathrm{O}^{ \pm}(4, q)$ space. (If $q=2$ then $\Omega$ spans an $\mathrm{O}^{-}(4,2)$-space.)

Lemma B.1. Every hyperplane meets $\Omega$. More precisely, there are exactly five types of hyperplanes $H$ of $U$ :

(i) Tangent hyperplanes $p^{\perp}$ for $p \in \Omega$, with $r \in H$ and $H \cap \Omega=\{p\}$;

(ii) Secant hyperplanes $x^{\perp}=H$ containing $r$, where $x$ is a singular point, $x^{\perp} \cap \Omega$ is a circle of $\Im(\Omega)$ and $\left\langle x^{\perp} \cap \Omega\right\rangle=x^{\perp}$;

(iii) $\mathrm{O}^{-}(4, q)$-hyperplanes for which $H \cap \Omega$ is an orbit of a cyclic subgroup of $G$ of order $|H \cap \Omega|=q \pm \sqrt{2 q}+1$ acting irreducibly on $U / r$; and

(iv) $\mathrm{O}^{+}(4, q)$-hyperplanes for which $H \cap \Omega$ contains an orbit of a cyclic subgroup of $G$ of order $|H \cap \Omega|-2=q-1$ that fixes two points of $H \cap \Omega$. Moreover, $H \cap \Omega$ is not one of the circles in (ii).

Proof. (i) Projecting mod $r$ shows that each point of $\Omega$ behaves as stated.

(ii) If $x$ is a singular point not in $\Omega$ then each of the $q+1$ t.s. lines on $x$ meets $\Omega$ since $\Omega$ is an ovoid, so that $\left|x^{\perp} \cap \Omega\right|=q+1$. Also, $\operatorname{dim}\left\langle x^{\perp} \cap \Omega\right\rangle=4$, as otherwise its set of singular points would project into a plane of $U / r$, and hence be contained in a conic, which is not the case since $q>2$ [Ti3, pp. 51-52]. Since $\left\langle x^{\perp} \cap \Omega\right\rangle$ lies in the 4 -space $x^{\perp}$, these subspaces coincide

(iii) This is [BS2, Theorem 1(a)]. 
(iv) The set of singular points of $H$ is partitioned by $q+1$ t.s. lines, and each t.s. line of $U$ meets $\Omega$ since $\Omega$ is an ovoid. Thus, $|H \cap \Omega|=q+1$.

We use the orbits of $G$ to find $G_{H}$. There are exactly two point-orbits on $U / r: \bar{\Omega}$ and the remaining $q\left(q^{2}+1\right)$ points. There is a subgroup of $G$ of order $q-1$ that fixes four points of $U / r$ and induces all scalars on each of these 1-spaces [HB, p. 183]. Since each line containing $r$ has a unique singular point, the two point-orbits on $U / r$ produce four point-orbits on $U-\{r\}$.

Since $G$ has five point-orbits it also has five hyperplane-orbits, so that all $q^{2}\left(q^{2}+\right.$ 1) $/ 2$ hyperplanes $H$ in (iv) lie in an orbit. Then $\left|G_{H}\right|=|G| /\left[q^{2}\left(q^{2}+1\right) / 2\right]=2(q-1)$, so that $G_{H}$ is dihedral of order $2(q-1)$, with orbits of size 2 and $q-1$ on $\Omega \mathrm{Su}$, Theorem 9].

For the final assertion, if $H \cap \Omega$ lies in two hyperplanes then it is in a plane, and hence is a conic, which is not the case [Ti3, pp. 51-52].

U. of Oregon, Eugene, OR 97403 and Northeastern U., Boston, MA 02115

E-mail address: kantor@uoregon.edu 